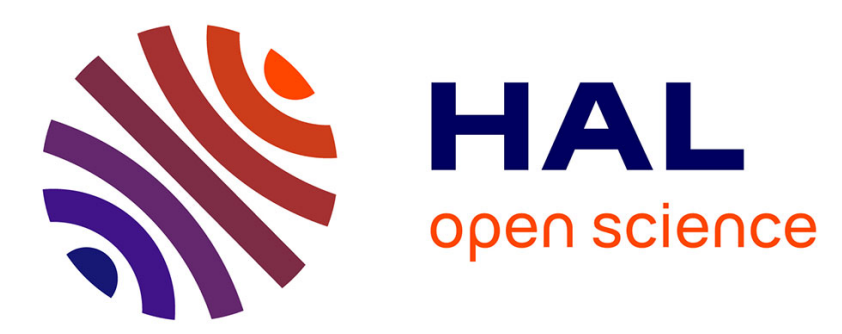

\title{
Asymptotics of linear initial boundary value problems with periodic boundary data on the half-line and finite intervals
}

Guillaume Dujardin

\section{- To cite this version:}

Guillaume Dujardin. Asymptotics of linear initial boundary value problems with periodic boundary data on the half-line and finite intervals. Proceedings of the Royal Society A: Mathematical, Physical and Engineering Sciences, 2009, 465, pp.3341-3360. 10.1098/rspa.2009.0194 . hal-00782033

\section{HAL Id: hal-00782033 \\ https://hal.science/hal-00782033}

Submitted on 28 Jan 2013

HAL is a multi-disciplinary open access archive for the deposit and dissemination of scientific research documents, whether they are published or not. The documents may come from teaching and research institutions in France or abroad, or from public or private research centers.
L'archive ouverte pluridisciplinaire HAL, est destinée au dépôt et à la diffusion de documents scientifiques de niveau recherche, publiés ou non, émanant des établissements d'enseignement et de recherche français ou étrangers, des laboratoires publics ou privés. 


\title{
Asymptotics of linear initial boundary value problems with periodic boundary data on the half-line and finite intervals
}

\author{
Guillaume Dujardin
}

10th April 2009

\begin{abstract}
This paper deals with the asymptotic behaviour of the solutions of linear initial boundary value problems with constant coefficients on the half-line and on finite intervals. We assume that the boundary data are periodic in time and we investigate whether the solution becomes time-periodic after sufficiently long time. Using the Fokas' transformation method, we show that for the linear Schrödinger equation, the linear heat equation and the linearised KdV equation on the half-line, the solutions indeed become periodic for large time. However, for the same linear Schrödinger equation on a finite interval, we show that the solution, in general, is not asymptotically periodic; actually, the asymptotic behaviour of the solution depends on the commensurability of the time period $T$ of the boundary data with the square of the length of the interval over $\pi$.
\end{abstract}

\section{Introduction}

This paper deals with the asymptotic behaviour of the solutions of linear initial boundary value problems (IBVP) with constant coefficients on the half-line and on finite intervals when the boundary data are periodic. Following the recent investigation of Bona and Fokas [4], our main concern is the existence of a periodic asymptotic profile for the solutions of such problems. As we shall see, the situation is rather different on the half-line and on finite intervals, because in the case of a finite interval, the "waves" can be "reflected" from one boundary to the other.

We first look at linear problems on the half-line written in the form

$$
\begin{cases}\partial_{t} q+\omega\left(-i \partial_{x}\right) q=0 & t>0, \quad x>0 \\ q(0, x)=q_{0}(x) & x>0 \\ \partial_{x}^{j} q(t, 0)=f_{j}(t) & t>0, \quad 0 \leq j \leq N-1,\end{cases}
$$


where $\omega$ is a polynomial of degree $n \in \mathbb{N}^{\star}, q_{0}$ is a smooth initial datum, $N \in$ $\{1, \ldots, n\}$ is the number of boundary data such that the problem is well-posed (see [10]), and $\left(f_{j}\right)_{0 \leq j \leq N-1}$ are $N$ given smooth functions that are compatible with $q_{0}$. We assume that $f_{0}, \ldots, f_{N-1}$ are periodic functions of time with the same period and we look for a smooth asymptotic profile $q_{p}$ such that

- for all $x \geq 0, t \mapsto q_{p}(t, x)$ is a periodic function of time,

- for all $x \geq 0,\left|q(t, x)-q_{p}(t, x)\right| \underset{t \rightarrow+\infty}{\longrightarrow} 0$.

In [4], the existence of a periodic profile for the solution of the linear KdV equation on the half-line (corresponding to $\left.\omega\left(-i \partial_{x}\right)=-i k^{3}\right)$ is stated, provided that the initial datum $q_{0}$ is homogeneous $\left(q_{0} \equiv 0\right)$ and that the boundary datum $f_{0}$ is periodic ( $N=1$ in that case). The proof is based on the new general transform method developped by A.S. Fokas [6]. In this paper, we use the Fokas' method to derive the asymptotic behaviour of the solutions of such equations with periodic boundary data. More precisely, for the solutions of the linear Schrödinger equation, of the linear heat equation and of the linear KdV equation on the halfline, we prove the existence of an asymptotic periodic profile when the periodic boundary data are periodic and we provide explicit formulae for these profiles involving the Fourier coefficients of the boundary data.

We also investigate the asymptotic behaviour of the solutions of linear PDEs with constant coefficients on bounded intervals with periodic boundary data of the form:

$$
\begin{cases}\partial_{t} q+\omega\left(-i \partial_{x}\right) q=0 & t>0, \quad x \in(0, L) \\ q(0, x)=q_{0}(x) & x \in(0, L) \\ \partial_{x}^{j} q(t, 0)=f_{j}(t) & t>0, \quad 0 \leq j \leq N_{1}-1 \\ \partial_{x}^{j} q(t, L)=g_{j}(t) & t>0, \quad 0 \leq j \leq N_{2}-1,\end{cases}
$$

where $L>0$ is given, $\omega$ is a polynomial of degree $n \in \mathbb{N}^{\star}, q_{0}$ is a smooth initial datum, $N_{1}, N_{2} \in\{1, \ldots, n\}$ are the numbers of data at $x=0$ and $x=L$ respectively such that the problem is well-posed (see [9], Appendix A, and [12]), and $\left(f_{j}\right)_{0 \leq j \leq N_{1}-1}$ and $\left(g_{j}\right)_{0 \leq j \leq N_{2}-1}$ are $N_{1}+N_{2}$ given smooth functions that are compatible with $q_{0}$. We assume that $f_{0}, \ldots, f_{N_{1}-1}$ and $g_{0}, \ldots, g_{N_{2}-1}$ are periodic functions of time with the same period and we look for a smooth asymptotic profile $q_{p}$ such that

- for all $x \in[0, L], t \mapsto q_{p}(t, x)$ is a periodic function of time,

- for all $x \in[0, L],\left|q(t, x)-q_{p}(t, x)\right| \underset{t \rightarrow+\infty}{\longrightarrow} 0$.

In the case of the linear Schrödinger equation, we use the formula derived in [9] to obtain results on the long-time behaviour of the solution. In particular, this formula allows us to give sufficient conditions depending on the link between the length $L$ of the interval and the period $T$ of the boundary data to obtain periodic solutions, as well as solutions that do not have any asymptotic profile. 
The outline of the paper is the following. In Section 2, we consider the linear Schrödinger equation on the half-line (i.e. a problem of the form (1.1) with $\left.\omega(k)=i k^{2}\right)$ with homogeneous initial datum $\left(q_{0} \equiv 0\right)$ and smooth periodic boundary datum $f_{0}$. We recall the Fokas' method (see [8]), we prove the existence of a periodic profile $q_{p}$ and we provide an explicit formula for that profile involving the Fourier coefficients of $f_{0}$ (see Theorem 2.4, Theorem 2.7 and Theorem 2.14). Section 3 is devoted to another illustration of the efficiency of the integral representation method for the analysis of linear IBVP on the half-line with periodic boundary data through two examples : the linear heat equation $\left(\omega(k)=k^{2}\right)$ and the linearised $\mathrm{KdV}$ equation $\left(\omega(k)=-i k^{3}\right)$. In both cases, we follow the method we used in Section 2: we use the integral representation method, we prove the existence of an asymptotic profile for the exact solution of the problem and we provide explicit formula for these profiles (see Theorem 3.1, Theorem 3.5 and Theorem 3.6). In Section 4, we consider problems of the form $(1.2)$ on a finite interval $(0, L)$ with $\omega(k)=i k^{2}$. If $f_{0}$ and $g_{0}$ are $T$-periodic smooth functions, we show that, if $L^{2} / \pi$ and $T$ are linearly dependent on $\mathbb{Q}$, the solution of these linear Schrödinger equations can have, for example, unbounded $L^{2}$-norm $\left(\|q(t)\|_{L^{2}(0, L)} \underset{t \rightarrow+\infty}{\longrightarrow}+\infty\right)$ and hence they cannot be asymptotically periodic in general. If $T$ and $L^{2} / \pi$ are linearly independent on $\mathbb{Q}$, we show that the solution is not asymptotically periodic in general as well. Finally, we provide numerical experiments in Section 5 for illustration.

\section{The linear Schrödinger equation on the half-line}

In this section, we consider the linear Schrödinger equation on the half-line with a periodic boundary datum and we apply the Fokas' method (see [8]). We first look at the asymptotic behaviour of the solution when the boundary datum is a sine function, we prove the existence of a periodic profile in that case and give an explicit formula for that profile using contour deformations of the involved integrals and Cauchy's residue theorem. Then, we apply the same method to the case of a general periodic boundary datum. We prove the existence of a periodic profile in that case and provide an explicit formula for that profile involving the Fourier coefficients of the boundary datum. Recall that we provide numerical experiments in Section 5 for illustration.

\subsection{The problem}

Following [4], we consider the linear Schrödinger equation on the half-line written in the form of the inital-boundary-value problem:

$$
\left\{\begin{array}{rlrl}
i \partial_{t} q+\partial_{x x} q & =0 & t>0, x>0 \\
q(0, x) & =0 & & x>0 \\
q(t, 0) & =f_{0}(t) & t>0 .
\end{array}\right.
$$


This problem is of the form (1.1) with $\omega(k)=i k^{2}$. Note that $N=1$ in that case. Since the problem is linear, we restrict ourselves to the case $q_{0} \equiv 0$. Moreover, we assume that $f_{0}$ is a smooth periodic function of time.

\subsection{The Fokas' method}

We apply the method described in [4] to Problem (2.1). Our goal is to prove the integral representation formula (2.8) of the solution $q$ in terms of the boundary datum $f_{0}$.

\subsubsection{Step 1: Write (2.1) into a divergence form}

The first step of the method consists in rewriting the evolution partial differential equation in (2.1) as a family of linear partial differential equations in divergence form. In order to do that, we define for $t>0, x>0$ and $k \in \mathbb{C}$

$$
X(t, x, k):=-k q(t, x)+i \partial_{x} q(t, x),
$$

where $q$ denotes the exact solution of Problem (2.1). Then we derive that for all $t>0, x>0$ and $k \in \mathbb{C}$,

$$
\partial_{t}\left[e^{-i k x+\omega(k) t} q\right]-\partial_{x}\left[e^{-i k x+\omega(k) t} X(t, x, k)\right]=0 .
$$

\subsubsection{Step 2: Obtain a global relation from (2.2)}

The next step of the method consists in deriving a global relation involving transforms of the boundary datum $f_{0}(t)=q(t, 0)$ and the Neumann derivative of the solution at the boundary $\partial_{x} q(t, 0)$. In order to do that, we consider the Fourier transform of the solution of Problem (2.1): for all $t>0$ and all $k \in \mathbb{C}$ such that $\operatorname{Im}(k) \leq 0$, we define

$$
\hat{q}(t, k)=\int_{0}^{+\infty} q(t, x) e^{-i k x} \mathrm{~d} x,
$$

and for all $t>0$ and all $\kappa \in \mathbb{C}$,

$$
\tilde{f}_{0}(t, \kappa)=\int_{0}^{t} e^{\kappa s} q(s, 0) \mathrm{d} s \quad \text { and } \quad \tilde{f}_{1}(t, \kappa)=\int_{0}^{t} e^{\kappa s} \partial_{x} q(s, 0) \mathrm{d} s .
$$

Using the divergence form (2.2) of Problem (2.1), we obtain the following global relation: for all $t>0$ and all $k \in \mathbb{C}$ such that $\operatorname{Im}(k) \leq 0$,

$$
e^{\omega(k) t} \hat{q}(t, k)=k \tilde{f}_{0}(t, \omega(k))-i \tilde{f}_{1}(t, \omega(k)) .
$$

\subsubsection{Step 3: Inverse the global relation (2.4)}

Assuming sufficient smoothness and decay on the exact solution $q$ of the Problem (2.1), we derive from (2.4) using the inverse Fourier transform that

$$
\forall t, x>0, \quad q(t, x)=\frac{1}{2 \pi} \int_{-\infty}^{+\infty} e^{i k x-\omega(k) t}\left(k \tilde{f}_{0}(t, \omega(k))-i \tilde{f}_{1}(t, \omega(k))\right) \mathrm{d} k .
$$




\subsubsection{Step 4: Eliminate unknown boundary conditions}

Following [4], we denote

$$
D=\{k \in \mathbb{C} \quad \mid \operatorname{Re}(\omega(k))<0\},
$$

and we note that for all $z \in \mathbb{C}$, we have

$$
z \in D \Longleftrightarrow-z \in D
$$

Therefore, we use the transformation $z \mapsto-z$ in $(2.4)$ to get that

$$
\forall k \in \mathbb{R}, \forall t>0, \quad e^{\omega(k) t} \hat{q}(t,-k)=-k \tilde{f}_{0}(t, \omega(k))-i \tilde{f}_{1}(t, \omega(k)) .
$$

This yields

$$
\forall k \in \mathbb{R}, \forall t>0, \quad \tilde{f}_{1}(t, \omega(k))=i e^{\omega(k) t} \frac{\hat{q}(t, k)+\hat{q}(t,-k)}{2} .
$$

Hence, using Jordan's lemma, we have

$$
\forall t, x>0, \quad \frac{1}{2 i \pi} \int_{-\infty}^{+\infty} e^{i k x-\omega(k) t} \tilde{f}_{1}(t, \omega(k)) \mathrm{d} k=\frac{1}{2} q(t, x) .
$$

Eventually, plugging this result into the integral representation of the solution formula (2.5) yields

$$
\forall t, x>0, \quad q(t, x)=\frac{1}{2 \pi} \int_{-\infty}^{+\infty} 2 k e^{i k x-i k^{2} t} \tilde{f}_{0}(t, \omega(k)) \mathrm{d} k .
$$

\subsubsection{The integral representation of the solution of (2.1)}

In order to sum up our previous calculations, we state the following:

Theorem 2.1 Assume $f_{0}$ is a given smooth periodic function of time. Then the solution $q$ of the corresponding problem (2.1) is given by formula (2.8).

The remaining parts of this section are devoted to the use of the integral representation formula (2.8) for the description of the asymptotic behaviour of $q$. In Section 2.3, we look at the asymptotics when the boundary datum is a sine function. Then we look at the long time behaviour when the function $f_{0}$ is any smooth periodic function in Section 2.4.

\subsection{Sine functions as boundary data for Problem (2.1)}

Inspired by [5], we look at the exact solution $q$ of Problem (2.1) with the simple periodic boundary datum:

$$
f_{0}(t)=\sin (b t)
$$


for some $b>0$. We are interested in the long time behaviour of the function $q$. Our goal is to prove Theorem 2.4 that provides an explicit formula for the asymptotic profile of $q$.

Using the definition of the time-transform of the boundary datum $f_{0}$ (see (2.3)), we derive that

$$
\tilde{f}_{0}(t, k)=\frac{b+k e^{k t} \sin (b t)-b e^{k t} \cos (b t)}{k^{2}+b^{2}} .
$$

We split the corresponding integrand in the integral representation formula (2.8) in the following way. We define for all $t, x>0$ and all $k \in \mathbb{C}$ such that $b^{2}-k^{4} \neq 0$,

$$
F_{1}(t, x, k)=2 k e^{i k x} \frac{b e^{-i k^{2} t}}{b^{2}-k^{4}}
$$

and

$$
F_{2}(t, x, k)=2 k e^{i k x} \frac{i k^{2} \sin (b t)-b \cos (b t)}{b^{2}-k^{4}} .
$$

This way, we have

$$
2 k e^{i k x} e^{-\omega(k) t} \tilde{f}_{0}(t, \omega(k))=F_{1}(t, x, k)+F_{2}(t, x, k) .
$$

Note that that $k \mapsto F_{1}(t, x, k)+F_{2}(t, x, k)$ is a holomorphic function on $\mathbb{C}$ (singularities in $\pm i \sqrt{|b|}$ and $\pm \sqrt{|b|}$ are removable), but $k \mapsto F_{1}(t, x, k)$ and $k \mapsto F_{1}(t, x, k)$ have singularities in $\pm i \sqrt{|b|}$ and $\pm \sqrt{|b|}$.

Now, we perform two contour deformations on the integral (2.8) in order to derive the asymptotic behaviour of $q$. Firstly, we define the following complex paths:

$$
\begin{aligned}
\forall r<0, & \Gamma_{1}(r)=r e^{-i \frac{\pi}{4}}, \\
\forall \theta \in(-\pi, 0), & \Gamma_{2}(\theta)=\sqrt{b}\left(1+e^{i \theta}\right), \\
\forall r>0, & \Gamma_{3}(r)=2 \sqrt{b}+r .
\end{aligned}
$$

This way, the exact solution of Problem (2.1) reads, after contour deformation in formula (2.8)

$$
\begin{aligned}
& q(t, x)= \\
& \quad \frac{1}{2 \pi} \int_{-\infty}^{0}\left(F_{1}\left(t, x, \Gamma_{1}(r)\right)+F_{2}\left(t, x, \Gamma_{1}(r)\right)\right) e^{-i \frac{\pi}{4}} \mathrm{~d} r \\
& +\frac{1}{2 \pi} \int_{-\pi}^{0}\left(F_{1}\left(t, x, \Gamma_{2}(\theta)\right)+F_{2}\left(t, x, \Gamma_{2}(\theta)\right)\right) i \sqrt{b} e^{i \theta} \mathrm{d} \theta \\
& +\frac{1}{2 \pi} \int_{0}^{+\infty}\left(F_{1}\left(t, x, \Gamma_{3}(r)\right)+F_{2}\left(t, x, \Gamma_{3}(r)\right)\right) \mathrm{d} r .
\end{aligned}
$$

Secondly, we use the two following complex paths

$$
\begin{array}{ll}
\forall r>0, & \Gamma_{3}^{+}(r)=2 \sqrt{b}+r e^{i \frac{\pi}{4}}, \\
\forall r>0, & \Gamma_{3}^{-}(r)=2 \sqrt{b}+r e^{-i \frac{\pi}{4}},
\end{array}
$$


to derive that

$$
\begin{aligned}
\frac{1}{2 \pi} \int_{0}^{+\infty} F_{1}\left(t, x, \Gamma_{3}(r)\right) \mathrm{d} r & =\frac{1}{2 \pi} \int_{0}^{+\infty} F_{1}\left(t, x, \Gamma_{3}^{-}(r)\right) e^{-i \frac{\pi}{4}} \mathrm{~d} r, \\
\frac{1}{2 \pi} \int_{0}^{+\infty} F_{2}\left(t, x, \Gamma_{3}(r)\right) \mathrm{d} r & =\frac{1}{2 \pi} \int_{0}^{+\infty} F_{2}\left(t, x, \Gamma_{3}^{+}(r)\right) e^{i \frac{\pi}{4}} \mathrm{~d} r
\end{aligned}
$$

and we can plug these formulae in the last line of (2.10) to get another expression for the exact solution $q$ of Problem (2.1).

This way of writing the exact solution, though it is maybe not the simplest one, has two important features. First of all, it decouples the quantities that have different behaviours (some of them tend to 0 when $t$ tends to $+\infty$ (See Lemma 2.2) whereas the others carry the information about the profile of the solution when $t$ tends to $+\infty$ (See Lemma 2.3)). Second of all, the integrals involved in this expression of the exact solution are suitable for numerical computations since they are either integrals of smooth functions over a finite interval, either integrals of smooth exponentially decaying functions over a half-line.

A precise statement on the asymptotic behaviour of the solution $q(t, x)$ of Problem (2.1) with boundary datum (2.9) when $x>0$ is given and $t$ tends to $+\infty$ will be derived from the two following lemmas.

Lemma 2.2 For all $x>0$, the following limits hold:

$$
\begin{aligned}
& \int_{-\infty}^{0} F_{1}\left(t, x, \Gamma_{1}(r)\right) e^{-i \frac{\pi}{4}} \mathrm{~d} r \underset{t \rightarrow+\infty}{\longrightarrow} 0, \\
& \int_{-\pi}^{0} F_{1}\left(t, x, \Gamma_{2}(\theta)\right) i \sqrt{b} e^{i \theta} \mathrm{d} \theta \underset{t \rightarrow+\infty}{\longrightarrow} 0, \\
& \int_{0}^{+\infty} F_{1}\left(t, x, \Gamma_{3}^{-}(r)\right) e^{-i \frac{\pi}{4}} \mathrm{~d} r \underset{t \rightarrow+\infty}{\longrightarrow} 0 .
\end{aligned}
$$

Lemma 2.3 The residue theorem ensures that

$$
\frac{1}{2 i \pi} \int_{\Gamma_{1}+\Gamma_{2}+\Gamma_{3}^{+}} F_{2}(t, x, k) \mathrm{d} k=\frac{e^{i x \sqrt{b}} e^{-i b t}-e^{-x \sqrt{b}} e^{i b t}}{2} .
$$

Proof. For all $t, x>0$, we have

$$
(k-\sqrt{b}) F_{2}(t, x, k)=2 k e^{i k x} \frac{b \cos (b t)-i k^{2} \sin (b t)}{\left(k^{2}+b\right)(k+\sqrt{b})} \underset{k \rightarrow \sqrt{b}}{\longrightarrow} \frac{e^{i x \sqrt{b}} e^{-i b t}}{2},
$$

and similarly,

$$
(k-i \sqrt{b}) F_{2}(t, x, k)=2 k e^{i k x} \frac{b \cos (b t)-i k^{2} \sin (b t)}{\left(k^{2}-b\right)(k+i \sqrt{b})} \underset{k \rightarrow i \sqrt{b}}{\longrightarrow}-\frac{e^{-x \sqrt{b}} e^{i b t}}{2} .
$$

Finally, we derive the following result on the asymtpotic behaviour of the solution $q$ of problem (2.1) with boundary datum (2.9): 
Theorem 2.4 For all $b>0$, let $q$ be the solution of Problem (2.1) with boundary datum (2.9). The following estimate holds for all $x>0$ :

$$
q(t, x) \underset{t \rightarrow+\infty}{\sim} \frac{i e^{i x \sqrt{b}} e^{-i b t}-i e^{-x \sqrt{b}} e^{i b t}}{2} .
$$

Proof. Use the results of Lemma 2.2 and Lemma 2.3 in expression (2.10).

\subsection{General periodic boundary data}

In this section, we look at the asymptotic behaviour of the solution $q$ of Problem (2.1) when the boundary datum $f_{0}$ is a smooth periodic function of time. Our goal is to prove Theorem 2.7 and Theorem 2.14 that prove the existence of a periodic profile for $q$ and provide explicit formulae for that profile involving the Fourier coefficients of $f_{0}$.

\subsubsection{Zero-mean periodic boundary data}

Since Equation (2.1) is linear and posed on the half-line, we can assume without loss of generality that the periodic boundary datum $f_{0}$ has period $2 \pi$. Since $f_{0}$ is smooth, we write

$$
\forall t \in \mathbb{R}, \quad f_{0}(t)=\sum_{n \in \mathbb{Z}} \hat{f}_{n} e^{i n t}
$$

where the Fourier coefficients $\left(\hat{f}_{n}\right)_{n \in \mathbb{Z}}$ tend to 0 rapidly as $|n|$ tends to $+\infty$.

We assume that $f_{0}$ is compatible with the homogeneous initial datum at $t=0$. In other words, we assume that

$$
f_{0}(0)=\sum_{n \in \mathbb{Z}} \hat{f}_{n}=0
$$

Moreover, we assume that $f_{0}$ has zero mean-value:

$$
\int_{0}^{2 \pi} f_{0}(t) \mathrm{d} t=2 \pi \hat{f}_{0}=0
$$

With these hypotheses on the boundary datum $g_{0}$ and following the method introduced in Section 2.3, we rewrite the exact solution $q$ of Problem (2.1) (see Lemma 2.5). We first define the three complex paths $\gamma_{1}, \gamma_{2}$ and $\gamma_{3}$ as follows:

$$
\begin{array}{rlrl}
\forall r & \in(-\infty, \sqrt{2}), & & \gamma_{1}(r)=r e^{-i \frac{\pi}{4}} \\
\forall r \in(1,+\infty), & & \gamma_{2}(r)=-i+r \\
\forall r \in(\sqrt{2},+\infty), & & \gamma_{3}(r)=r e^{-i \frac{\pi}{4}} .
\end{array}
$$

Lemma 2.5 Let $q$ be the exact solution of Problem (2.1) with $2 \pi$-periodic smooth boundary datum $f_{0}$ written in the form (2.12) and satisfying (2.13) and (2.14). The following identity holds for all $t, x>0$ :

$$
q(t, x)=\frac{1}{2 i \pi} \sum_{n \in \mathbb{Z}} \hat{f}_{n} \int_{\gamma_{1}+\gamma_{2}} 2 k e^{i k x} \frac{e^{i n t}-e^{-i k^{2} t}}{\left(n+k^{2}\right)} \mathrm{d} k .
$$


Remark 2.6 Note that for all $t, x>0$, every integrand is a smooth function of $k$ since the singularities (in $k= \pm \sqrt{-n}$ if $n \leq-1$ or in $k= \pm i \sqrt{n}$ if $n \geq 1$ ) are removable.

Theorem 2.7 We have the following asymptotic periodic profile for the exact solution of Problem (2.1) with smooth $2 \pi$-periodic boundary data $f_{0}$ written in the form (2.12) and satisfying (2.13) and (2.14): for all $x>0$,

$$
q(t, x) \underset{t \rightarrow+\infty}{\sim} \sum_{n=1}^{\infty}\left(\hat{f}_{n} e^{-x \sqrt{n}} e^{i n t}+\hat{f}_{-n} e^{i x \sqrt{n}} e^{-i n t}\right) .
$$

Proof. Using identity (2.17) of Lemma (2.5), we write that for all $n \in \mathbb{Z} \backslash\{0\}$,

$$
\begin{aligned}
& \int_{\gamma_{1}+\gamma_{2}} 2 k e^{i k x} \frac{e^{i n t}-e^{-i k^{2} t}}{\left(n+k^{2}\right)} \mathrm{d} k \\
& \quad=\int_{\gamma_{1}+\gamma_{2}} 2 k e^{i k x} \frac{e^{i n t}}{\left(n+k^{2}\right)} \mathrm{d} k-\int_{\gamma_{1}+\gamma_{2}} 2 k e^{i k x} \frac{e^{-i k^{2} t}}{\left(n+k^{2}\right)} \mathrm{d} k .
\end{aligned}
$$

Note that, by contour deformation,

$$
\int_{\gamma_{1}+\gamma_{2}} 2 k e^{i k x} \frac{e^{-i k^{2} t}}{\left(n+k^{2}\right)} \mathrm{d} k=\int_{\gamma_{1}+\gamma_{3}} 2 k e^{i k x} \frac{e^{-i k^{2} t}}{\left(n+k^{2}\right)} \mathrm{d} k,
$$

and for all $x>0$, this quantity tends to 0 when $t$ tends to $+\infty$, uniformly in $n$. Moreover, using Jordan's lemma, we get

$$
\frac{1}{2 i \pi} \int_{\gamma_{1}+\gamma_{2}} 2 k e^{i k x} \frac{e^{i n t}}{\left(n+k^{2}\right)} \mathrm{d} k= \begin{cases}e^{i x \sqrt{-n}} e^{i n t} & \text { if } n \leq-1 \\ e^{-x \sqrt{n}} e^{i n t} & \text { if } n \geq 1\end{cases}
$$

Remark 2.8 Note that, setting $b=1$ in (2.9), Theorem 2.7 implies

$$
\forall x>0, \quad q(t, x) \underset{t \rightarrow+\infty}{\sim}\left(\frac{1}{2 i} e^{-x} e^{i t}-\frac{1}{2 i} e^{i x} e^{-i t}\right) .
$$

This result is exactly the corresponding relation (2.11) of Theorem 2.4.

Remark 2.9 Formula (2.18) of Theorem 2.7 ensures that the Sobolev regularity (in time) of the asymptotic profile of the exact solution of Problem (2.1) is the same as that of the periodic datum $f_{0}$ (recall that $f_{0}$ is assumed sufficiently smooth anyway).

Remark 2.10 Further analysis of the convergence of $\int_{\gamma_{1}+\gamma_{3}} 2 k e^{i k x} \frac{e^{-i k^{2} t}}{\left(n+k^{2}\right)} \mathrm{d} k$ to 0 shows that the convergence in (2.18) is uniform in $x$ on bounded sets. 


\subsubsection{General periodic boundary data}

In order to get rid of the somehow artificial condition (2.14), we compute the limit as $t$ tends to $+\infty$ of the formal exact solution of Problem (2.1) obtained using formula (2.8) with boundary datum $f_{0}(t) \equiv 1$ in Lemma 2.12. Before that, we define the following complex paths (note that they are a shifted version of the family $\left(\gamma_{1}, \gamma_{2}, \gamma_{3}\right)$ defined in section 2.4.1):

$$
\begin{array}{lc}
\forall r \in(-\infty, 0), & \Gamma_{1}(r)=-i+r e^{-i \frac{\pi}{4}} \\
\forall r \in(0,+\infty), & \Gamma_{2}(r)=-i+r \\
\forall r \in(0,+\infty), & \Gamma_{3}(r)=-i+r e^{-i \frac{\pi}{4}},
\end{array}
$$

and provide us with another lemma:

Lemma 2.11 The following equality holds:

$$
\frac{1}{2 i \pi} \int_{\Gamma_{1}+\Gamma_{3}} \frac{e^{-i z^{2}}}{z} \mathrm{~d} z=\frac{1}{2}
$$

Proof. Using contour deformation, the left hand side of the identity reads

$$
\frac{1}{2 i \pi} \int_{-\infty}^{-1} \frac{e^{-i k^{2}}}{k} \mathrm{~d} k+\frac{1}{2 i \pi} \int_{-\pi}^{0} i e^{-i e^{2 i \theta}} \mathrm{d} \theta+\frac{1}{2 i \pi} \int_{1}^{+\infty} \frac{e^{-i k^{2}}}{k} \mathrm{~d} k .
$$

The left and the right term cancel out and the double of the last term is

$$
\frac{1}{2 i \pi} \int_{-\pi}^{0} i e^{-i e^{2 i \theta}} \mathrm{d} \theta+\frac{1}{2 i \pi} \int_{0}^{\pi} i e^{-i e^{2 i \theta}} \mathrm{d} \theta=\frac{1}{2 i \pi} \int_{\mathcal{C}} \frac{e^{-i k^{2}}}{k} \mathrm{~d} k,
$$

where $\mathcal{C}$ stands for the positive unit circle. Hence the result is proved using Cauchy's residue theorem:

$$
2\left(\frac{1}{2 i \pi} \int_{\Gamma_{1}+\Gamma_{3}} \frac{e^{-i z^{2}}}{z} \mathrm{~d} z\right)=\frac{1}{2 i \pi} \int_{\mathcal{C}} \frac{e^{-i k^{2}}}{k} \mathrm{~d} k=1 .
$$

We are now able to prove the result about the asymptotic behaviour of the formal exact solution of Problem (2.1) obtained using formula (2.8) with boundary datum $f_{0}(t) \equiv 1$ :

Lemma 2.12 For all $x>0$,

$$
\frac{1}{2 \pi} \int_{-\infty}^{+\infty} 2 k e^{i k x} e^{-i k^{2} t} \int_{0}^{t} e^{i k^{2} s} \mathrm{~d} s \mathrm{~d} k \underset{t \rightarrow+\infty}{\longrightarrow} 1
$$

Proof. Note that for all $t, x>0$, one has

$$
\frac{1}{2 \pi} \int_{-\infty}^{+\infty} 2 k e^{i k x} e^{-i k^{2} t} \int_{0}^{t} e^{i k^{2} s} \mathrm{~d} s \mathrm{~d} k=\frac{1}{2 i \pi} \int_{-\infty}^{+\infty} 2 e^{i k x} \frac{1-e^{-i k^{2} t}}{k} \mathrm{~d} k .
$$


Moreover, since the function $k \mapsto e^{i k x} \frac{1-e^{-i k^{2} t}}{k}$ is analytic, we have

$$
\int_{-\infty}^{+\infty} 2 e^{i k x} \frac{1-e^{-i k^{2} t}}{k} \mathrm{~d} k=\int_{\Gamma_{1}+\Gamma_{2}} 2 e^{i k x} \frac{1-e^{-i k^{2} t}}{k} \mathrm{~d} k .
$$

Using the linearity of the integral, we derive that

$$
\int_{-\infty}^{+\infty} 2 e^{i k x} \frac{1-e^{-i k^{2} t}}{k} \mathrm{~d} k=\int_{\Gamma_{1}+\Gamma_{2}} \frac{2 e^{i k x}}{k} \mathrm{~d} k-\int_{\Gamma_{1}+\Gamma_{2}} \frac{2 e^{i k x-i k^{2} t}}{k} \mathrm{~d} k .
$$

The quantity we want to compute is now the sum of two contributions. Using Jordan's lemma, we compute directly the first contribution:

$$
\frac{1}{2 i \pi} \int_{\Gamma_{1}+\Gamma_{2}} \frac{2 e^{i k x}}{k} \mathrm{~d} k=2 .
$$

Then, we compute the second contribution using another contour deformation:

$$
\int_{\Gamma_{1}+\Gamma_{2}} \frac{2 e^{i k x-i k^{2} t}}{k} \mathrm{~d} k=\int_{\Gamma_{1}+\Gamma_{3}} \frac{2 e^{i k x-i k^{2} t}}{k} \mathrm{~d} k .
$$

We have, setting $z=k \sqrt{t}$,

$$
\int_{\Gamma_{1}+\Gamma_{3}} \frac{e^{-i k^{2} t}}{k} e^{i k x} \mathrm{~d} k=\int_{\Gamma_{1}+\Gamma_{3}} \frac{e^{-i z^{2}}}{z} e^{i z \frac{x}{\sqrt{t}}} \mathrm{~d} z .
$$

Note that

$$
e^{i z \frac{x}{\sqrt{t}}} \underset{t \rightarrow+\infty}{\longrightarrow} 1
$$

and for all $t \geq 1$ and $r \in \mathbb{R}$,

$$
\left|\frac{e^{-i\left(r e^{i \frac{\pi}{4}}-i\right)^{2}}}{r e^{-i \frac{\pi}{4}}-i} e^{i\left(r e^{i \frac{\pi}{4}}-i\right) \frac{x}{\sqrt{t}}}\right| \leq \frac{e^{-2 \frac{\sqrt{2}}{2} r\left(1+r \frac{\sqrt{2}}{2}\right)}}{\left(\frac{1}{2}+\left(r+\frac{\sqrt{2}}{2}\right)^{2}\right)^{1 / 2}} e^{\max \left(0,1+\frac{\sqrt{2}}{2} r\right) x} .
$$

Since this bound is integrable on $\mathbb{R}$ and independent of $t \geq 1$, we derive that

$$
\int_{\Gamma_{1}+\Gamma_{3}} \frac{e^{-i z^{2}}}{z} e^{i z \frac{x}{\sqrt{t}}} \mathrm{~d} z \underset{t \rightarrow+\infty}{\longrightarrow} \int_{\Gamma_{1}+\Gamma_{3}} \frac{e^{-i z^{2}}}{z} \mathrm{~d} z .
$$

This last integral is equal to $i \pi$ by Lemma 2.11. Finally,

$$
\frac{1}{2 i \pi} \int_{\Gamma_{1}+\Gamma_{2}} \frac{2 e^{i k x-i k^{2} t}}{k} \mathrm{~d} k \underset{t \rightarrow+\infty}{\longrightarrow} 1,
$$

and the result is proved.

Remark 2.13 This lemma even allows us to consider boundary data $f_{0}$ that do not satisfy the compatibility condition (2.13).

Finally, we are able to use the linearity of the problem to combine Theorem 2.7 with Lemma 2.12 to obtain the following theorem: 
Theorem 2.14 Assume $f_{0}$ is a given sufficiently smooth $2 \pi$-periodic complexvalued function written in the form (2.12) and satisfying (2.13). We have the following result for the asymptotic behaviour of the exact solution $q$ of Problem (2.1): for all $x>0$,

$$
q(t, x) \underset{t \rightarrow+\infty}{\sim} \hat{f}_{0}+\sum_{n=1}^{\infty}\left(\hat{f}_{n} e^{-x \sqrt{n}} e^{i n t}+\hat{f}_{-n} e^{i x \sqrt{n}} e^{-i n t}\right) .
$$

Remark 2.15 This result gives an explicit description of the asymptotic profile of the exact solution of the linear Schrödinger equation on the half-line (2.1) for homogeneous initial datum and periodic boundary datum.

\section{The linear heat equation and the linear $\mathrm{KdV}$ equation on the half-line}

This section is devoted to another illustration of the efficiency of the Fokas' method for the analysis of the asymptotic behaviour of the solutions of linear initial boundary value problems on the half-line. As we did in Section 2, we apply this integral representation method to the solutions of linear evolution partial differential equations with constant coefficients of the form (1.1) on the halfline with periodic boundary data and we use contour deformation and Cauchy's residue theorem to derive the existence of periodic profiles and obtain explicit formulae involving the Fourier coefficients of the boundary data (see Theorem 3.1, Theorem 3.5 and Theorem 3.6). Since the method is very similar to the one used in the previous section (Section 2), the results are presented in a more concise form. We first look at the linear heat equation in Section 3.1. Then we investigate the case of the linear $\mathrm{KdV}$ equation in Section 3.2, providing an explicit description of the periodic profile whose existence was stated in [4].

\subsection{The linear heat equation on the half-line}

In this section, we investigate the long time behaviour of the solutions of the linear heat equation on the half-line with periodic boundary data. This problem is of the form (1.1) with $\omega(k)=k^{2}$. Using the linearity of the problem, we restrict ourselves to the case of homogeneous initial data $q_{0} \equiv 0$. Moreover, we assume that $f_{0}$ is a smooth function of $t$ and is $2 \pi$-periodic.

\subsubsection{The transformation method}

We are interested in the long time behaviour of the solution of the following problem:

$$
\left\{\begin{array}{rlrl}
\partial_{t} q-\partial_{x x} q & =0 & t>0, x>0 \\
q(0, x) & =0 & x>0 \\
q(t, 0) & =f_{0}(t) & t>0
\end{array}\right.
$$


where $f_{0}$ is a smooth $2 \pi$-periodic function. Applying the Fokas' transformation method (see Section 2.2), we obtain the following representation formula for the exact solution of Problem (3.1):

$$
\forall t, x>0, \quad q(t, x)=\frac{1}{2 i \pi} \int_{-\infty}^{+\infty} 2 k e^{i k x-k^{2} t} \tilde{f}_{0}(t, \omega(k)) \mathrm{d} k,
$$

where $\omega(k)=k^{2}$ and

$$
\tilde{f}_{0}(t, \kappa)=\int_{0}^{t} e^{\kappa s} f_{0}(s) \mathrm{d} s
$$

\subsubsection{Periodic boundary data with zero mean}

Theorem 3.1 We have the following asymptotic periodic profile for the exact solution of Problem (3.1) with smooth $2 \pi$-periodic boundary data $f_{0}$ written in the form (2.12) and satisfying (2.13) and (2.14): for all $x>0$,

$$
q(t, x) \underset{t \rightarrow+\infty}{\sim} \sum_{n=1}^{\infty} e^{-x \sqrt{n} \frac{\sqrt{2}}{2}}\left(\hat{f}_{n} e^{-i x \sqrt{n} \frac{\sqrt{2}}{2}} e^{i n t}+\hat{f}_{-n} e^{i x \sqrt{n} \frac{\sqrt{2}}{2}} e^{-i n t}\right) .
$$

Moreover, the convergence is uniform with respect to $x$.

Proof. By a straightforward calculation, we get, for $n \in \mathbb{Z} \backslash\{0\}$,

$$
\tilde{f}_{0}\left(t, k^{2}\right)=\frac{e^{i n t} e^{k^{2} t}-1}{k^{2}+i n} .
$$

Hence, defining for all $t, x>0$,

$$
F_{1}(t, x, k)=2 k e^{i k x} \frac{-e^{-k^{2} t}}{k^{2}+i n}
$$

and

$$
F_{2}(t, x, k)=2 k e^{i k x} \frac{e^{i n t}}{k^{2}+i n}
$$

we get,

$$
2 k e^{i k x} e^{-k^{2} t} \tilde{f}_{0}(t, \omega(k))=F_{1}(t, x, k)+F_{2}(t, x, k) .
$$

Using (3.2), we derive that for all $t, x>0$,

$$
q(t, x)=\frac{1}{2 i \pi} \int_{-\infty}^{+\infty} F_{1}(t, x, k) \mathrm{d} k+\frac{1}{2 i \pi} \int_{-\infty}^{+\infty} F_{2}(t, x, k) \mathrm{d} k .
$$

One easily checks that

$$
\frac{1}{2 i \pi} \int_{-\infty}^{+\infty} F_{1}(t, x, k) \mathrm{d} k \underset{t \rightarrow+\infty}{\longrightarrow} 0
$$

uniformly in $n$ and $x$. Moreover, using Jordan's lemma, we have

$$
\frac{1}{2 i \pi} \int_{-\infty}^{+\infty} F_{2}(t, x, k) \mathrm{d} k=e^{-x \sqrt{n} \frac{\sqrt{2}}{2}} e^{-i x \sqrt{n} \frac{\sqrt{2}}{2}} e^{i n t}
$$


if $n \geq 1$ and

$$
\frac{1}{2 i \pi} \int_{-\infty}^{+\infty} F_{2}(t, x, k) \mathrm{d} k=e^{-x \sqrt{-n} \frac{\sqrt{2}}{2}} e^{i x \sqrt{-n} \frac{\sqrt{2}}{2}} e^{i n t}
$$

if $n \leq-1$. This completes the proof.

Remark 3.2 One observes in Theorem 3.1 the following smoothing effect of the heat equation: for any periodic boundary datum $f_{0}$ with (sufficient) Sobolev-type regularity, for all $x>0$, the asymptotic profile of the exact solution of Problem (3.1) at point $x$ has a Gevrey-type regularity.

\subsubsection{General periodic boundary data}

As we did for the Schrödinger equation in Section 2.4, we investigate the asymptotic behaviour of the solution of Problem (3.1) with boundary datum $g_{0} \equiv 1$.

Lemma 3.3 Let $\Gamma$ be the complex path defined for $r \in \mathbb{R}$ by $\Gamma(r)=r-i$. One has

$$
\frac{1}{2 i \pi} \int_{\Gamma} \frac{e^{-z^{2}}}{z} \mathrm{~d} z=\frac{1}{2}
$$

Proof. The proof is similar to that of Lemma 2.11.

Lemma 3.4 For all $x>0$,

$$
\frac{1}{2 i \pi} \int_{-\infty}^{+\infty} 2 k e^{i k x} e^{-k^{2} t} \int_{0}^{t} e^{k^{2} s} \mathrm{~d} s \mathrm{~d} k \underset{t \rightarrow+\infty}{\longrightarrow} 1 .
$$

Proof. The proof is similar to that of Lemma 2.12. The above integral reads by contour deformation

$$
\frac{1}{2 i \pi} \int_{-\infty}^{+\infty} 2 e^{i k x} \frac{1-e^{-k^{2} t}}{k} \mathrm{~d} k=\frac{1}{2 i \pi} \int_{\Gamma} 2 e^{i k x} \frac{1-e^{-k^{2} t}}{k} \mathrm{~d} k,
$$

where $\Gamma$ is the complex path defined for $r \in \mathbb{R}$ by $\Gamma(r)=r-i$. Note that

$$
\frac{1}{2 i \pi} \int_{\Gamma} 2 \frac{e^{i k x}}{k} \mathrm{~d} k=2
$$

by Jordan's lemma and

$$
\frac{1}{2 i \pi} \int_{\Gamma} 2 e^{i k x} \frac{e^{-k^{2} t}}{k} \mathrm{~d} k \underset{t \rightarrow+\infty}{\longrightarrow} 1,
$$

by Lemma 3.3. This completes the proof.

These lemmas allow us to generalise Theorem 3.1 to the case of general periodic boundary conditions: 
Theorem 3.5 We have the following asymptotic expansion for the exact solution of Problem (3.1) with smooth $2 \pi$-periodic boundary data $f_{0}$ written in the form (2.12) and satisfying (2.13): For all $x>0$,

$$
q(t, x) \underset{t \rightarrow+\infty}{\sim} \hat{f}_{0}+\sum_{n=1}^{\infty} e^{-x \sqrt{n} \frac{\sqrt{2}}{2}}\left(\hat{f}_{n} e^{-i x \sqrt{n} \frac{\sqrt{2}}{2}} e^{i n t}+\hat{f}_{-n} e^{i x \sqrt{n} \frac{\sqrt{2}}{2}} e^{-i n t}\right) .
$$

Proof. The proof consists in adding Lemma (3.4) to Theorem (3.1) by linearity.

\subsection{The linearised $\mathrm{KdV}$ equation}

In this section, we investigate the long time behaviour of the solutions of the linear Korteweg-de Vries (KdV) equation on the half-line with periodic boundary data. This problem is of the form (1.1) with $\omega(k)=-i k^{3}$. Using the linearity of the problem, we restrict ourselves to the case of homogeneous initial data $q_{0} \equiv 0$. Moreover, we assume that $f_{0}$ is a smooth function of $t$ and is $2 \pi$-periodic.

\subsubsection{The transformation method}

We are interested in the long time behaviour of the solution of the following problem:

$$
\left\{\begin{array}{rlrl}
\partial_{t} q+\partial_{x x x} q & =0 & t>0, x>0 \\
q(0, x) & =0 & x>0 \\
q(t, 0) & =f_{0}(t) & t>0,
\end{array}\right.
$$

where $f_{0}$ is a smooth $2 \pi$-periodic function. Applying the Fokas' transformation method, we obtain the following representation formula for the exact solution of Problem (3.5):

$$
\forall t, x>0, \quad q(t, x)=\frac{1}{2 \pi} \int_{-\infty}^{+\infty}-3 k^{2} e^{i k x+i k^{3} t} \tilde{f}_{0}(t, \omega(k)) \mathrm{d} k,
$$

where $\omega(k)=-i k^{3}$ and

$$
\tilde{f}_{0}(t, \kappa)=\int_{0}^{t} e^{\kappa s} f_{0}(s) \mathrm{d} s
$$

\subsubsection{Periodic boundary data with zero mean}

We denote by $\alpha$ the complex number $e^{i \frac{2 \pi}{3}}$. We define the two following complex paths for $\theta \in\left(0, \frac{\pi}{3}\right)$ :

$$
\begin{array}{ll}
\forall r \in(-\infty, 0), & \Gamma_{1}(r)=r e^{-i\left(\frac{\pi}{3}-\theta\right)}, \\
\forall r \in(0,+\infty), & \Gamma_{2}(r)=r e^{i\left(\frac{\pi}{3}-\theta\right)} .
\end{array}
$$


Theorem 3.6 We have the following asymptotic expansion for the exact solution of Problem (3.5) with smooth $2 \pi$-periodic boundary data $f_{0}$ written in the form (2.12) and satisfying (2.13) and (2.14): for all $x>0$,

$$
q(t, x) \underset{t \rightarrow+\infty}{\sim} \sum_{n=1}^{\infty}\left(\hat{f}_{n} e^{i \alpha \sqrt[3]{n} x} e^{i n t}+\hat{f}_{-n} e^{-i \alpha^{2} \sqrt[3]{n} x} e^{-i n t}\right) .
$$

Remark 3.7 Note that for all $\lambda \in \mathbb{R}$, the complex conjugate of $e^{i \alpha \lambda}$ is $e^{-i \alpha^{2} \lambda}$. If the boundary datum $f_{0}$ is real valued then for all $n \in \mathbb{Z}, \overline{\hat{f}_{n}}=\hat{f}_{-n}$. Hence, the asymptotic profile given by formula (3.7) is real valued as well.

Remark 3.8 Using the definition of $\alpha$, relation (3.7) also reads

$$
q(t, x) \underset{t \rightarrow+\infty}{\sim} \sum_{n=1}^{\infty} e^{-\frac{\sqrt{3}}{2} \sqrt[3]{n} x}\left(\hat{f}_{n} e^{-i \frac{1}{2} \sqrt[3]{n} x} e^{i n t}+\hat{f}_{-n} e^{i \frac{1}{2} \sqrt[3]{n} x} e^{-i n t}\right) .
$$

Proof. By a straightforward calculation, we get, for $n \in \mathbb{Z} \backslash\{0\}$,

$$
\tilde{f}_{0}\left(t,-i k^{3}\right)=\frac{e^{i n t} e^{-i k^{3} t}-1}{i\left(n-k^{3}\right)} .
$$

Hence, defining for all $t, x>0$,

$$
F_{1}(t, x, k)=3 k^{2} e^{i k x} \frac{-e^{i k^{3} t}}{\left(k^{3}-n\right)}
$$

and

$$
F_{2}(t, x, k)=3 k^{2} e^{i k x} \frac{e^{i n t}}{\left(k^{3}-n\right)}
$$

we get,

$$
-3 i k^{2} e^{i k x} e^{i k^{3} t} \tilde{f}_{0}(t, \omega(k))=F_{1}(t, x, k)+F_{2}(t, x, k) .
$$

Note that for all $t, x>0, F_{1}+F_{2}$ is an analytic function of $k$. Using (3.6) and contour deformation, we derive that for all $t, x>0$,

$$
q(t, x)=\frac{1}{2 i \pi} \int_{\Gamma_{1}+\Gamma_{2}} F_{1}(t, x, k) \mathrm{d} k+\frac{1}{2 i \pi} \int_{\Gamma_{1}+\Gamma_{2}} F_{2}(t, x, k) \mathrm{d} k .
$$

One easily checks that

$$
\frac{1}{2 i \pi} \int_{\Gamma_{1}+\Gamma_{2}} F_{1}(t, x, k) \mathrm{d} k \underset{t \rightarrow+\infty}{\longrightarrow} 0
$$

uniformly in $n$ and $x$. Moreover, using Jordan's lemma, we have

$$
\frac{1}{2 i \pi} \int_{\Gamma_{1}+\Gamma_{2}} F_{2}(t, x, k) \mathrm{d} k=e^{i \alpha \sqrt[3]{n} x} e^{i n t},
$$

if $n \geq 1$ and

$$
\frac{1}{2 i \pi} \int_{\Gamma_{1}+\Gamma_{2}} F_{2}(t, x, k) \mathrm{d} k=e^{i \alpha^{2} \sqrt[3]{n} x} e^{i n t},
$$

if $n \leq-1$. This completes the proof. 


\section{IBVP over the finite interval}

\subsection{Introduction}

In this section, we consider linear evolution PDEs with constant coefficients on a finite interval $(0, L)$ where $L$ is a given positive real number. These problems are of the form (1.2). Such problems have been studied in [9] by implementing the Fokas' transformation method.

We are interested in the long time behaviour of the solutions of these problems when the boundary data are periodic in time. In particular, just as we did for problems on the half line in the previous sections (see Section 2 and Section 3), we investigate the existence of a periodic profile for the solutions of such problems. More precisely, if $q(t, x)$ denotes the solution of such a problem for $t \geq 0$ and $x \in[0, L]$, we define an asymptotic profile $q_{p}(t, x)$ as a smooth periodic function of $t$ satisfying for all $x \in[0, L]$

$$
\left|q(t, x)-q_{p}(t, x)\right| \underset{t \rightarrow+\infty}{\longrightarrow} 0 .
$$

With the linear Schrödinger equation as an example, we present in this section different types of asymptotic behaviours that occur when the boundary conditions are periodic and share the same period. In contrast to the case of linear problems on the half-line (see Theorem 2.14), it turns out that an asymptotic profile does not exist in general (see Theorem 4.1, Theorem 4.4 and Theorem 4.9).

\subsection{The linear Schrödinger equation}

We investigate the long time behaviour of the solution of the following homogenous linear initial boundary value problem :

$$
\left\{\begin{array}{rlc}
i \partial_{t} q+\partial_{x x} q & =0 & t>0, x \in(0, L) \\
q(0, x) & =0 & x \in(0, L) \\
q(t, 0) & =f_{0}(t) & t>0 \\
q(t, L) & =g_{0}(t) & t>0,
\end{array}\right.
$$

where $L$ and $T$ are given positive real numbers and $f_{0}$ and $g_{0}$ are given smooth $T$-periodic functions. Note that this problem is of the form (1.2) with $\omega(k)=i k^{2}$ and $N_{1}=N_{2}=1$. Our analysis is based on the following classical representation formula of the solution of (4.1) (see [9], equation (3.2)): for all $t>0$ and $x \in$ $(0, L)$

$$
q(t, x)=\frac{i}{4 L} \sum_{m \in \mathbb{Z}} \sin \left(k_{m} x\right) e^{-i k_{m}^{2} t}\left[N\left(t, k_{m}\right)-N\left(t,-k_{m}\right)\right], \quad k_{m}=\frac{m \pi}{L},
$$

where the function $N$ is given by

$$
N(t, k)=k\left(\tilde{f}_{0}(t, k)-e^{-i k L} \tilde{g}_{0}(t, k)\right),
$$


and the functions $\tilde{f}_{0}$ and $\tilde{g}_{0}$ are the following $t$-transforms of $f_{0}$ and $g_{0}$ : for all $k \in \mathbb{C}$ and $t \geq 0$,

$$
\tilde{f}_{0}(t, k)=\int_{0}^{t} e^{i k^{2} s} f_{0}(s) \mathrm{d} s \quad \text { and } \quad \tilde{g}_{0}(t, k)=\int_{0}^{t} e^{i k^{2} s} g_{0}(s) \mathrm{d} s .
$$

Although the exact solution of Problem (4.1) is a smooth function of $(t, x)$, the representation formula (4.2) involves a series whose convergence can be weak (and is usually not uniform on $(0, L)$ for example). However, as an exact representation of the solution in terms of transforms of the boundary data, it provides much information in particular about the long time behaviour.

It turns out that the typical behaviour of the exact solution $q$ of (4.1) depends on whether $T$ and $L^{2} / \pi$ are linearly independent on $\mathbb{Q}$ or not. As we shall see, this condition corresponds to the possibility for a periodic signal of frequency $2 \pi / T$ given at a boundary to get reflected on the other boundary in a specific way. Subsection 4.3 is devoted to the case $T$ and $L^{2} / \pi$ are $\mathbb{Q}$-linearly dependent. Subsection 4.4 is devoted to the case $T$ and $L^{2} / \pi$ are $\mathbb{Q}$-linearly independent.

In the following, we set $b=2 \pi / T$. Moreover, for all measurable complex functions $h$ defined on $(0, L)$, we define

$$
\|h\|_{L^{2}(0, L)}=\left(\frac{1}{L} \int_{0}^{L}|h(x)|^{2} \mathrm{~d} \lambda(x)\right)^{1 / 2}
$$

and

$$
\|h\|_{L^{\infty}(0, L)}=\inf \{y \in \mathbb{R} \mid \lambda(\{x \in(0, L)|| h(x) \mid>y\})=0\},
$$

where $\lambda$ denotes the Lebesgue measure on $\mathbb{R}$. Of course, $L^{2}(0, L)$ (respectively $\left.L^{\infty}(0, L)\right)$ denotes the space of (classes of) complex functions $h$ on $(0, L)$ such that $\|h\|_{L^{2}(0, L)}<+\infty$ (resp. $\left.\|h\|_{L^{\infty}(0, L)}<+\infty\right)$.

\subsection{The dependent case}

\subsubsection{A simple example}

Before dealing with the general dependent case, we investigate a simple example. In this subsection, we assume that $T$ and $L^{2} / \pi$ are linearly dependent on $\mathbb{Q}$ in the following way:

$$
\frac{2}{T}=\frac{\pi}{L^{2}}
$$

Hence, we have $b=\pi^{2} / L^{2}$. Moreover, we take as boundary data in Problem (4.1) the following functions:

$$
f_{0}(t)=\sin (b t) \quad \text { and } \quad g_{0}(t)=0,
$$

for all $t \in \mathbb{R}$. Our result on the long time behaviour of $q$ in that case is the following: 
Theorem 4.1 Assume the period $T$ of the boundary conditions $f_{0}$ and $g_{0}$ defined in (4.5) satisfies relation (4.4). Then the exact solution of the linear Schrödinger equation (4.1) on $(0, L)$ satisfies

$$
\|q(t)\|_{L^{\infty}(0, L)} \underset{t \rightarrow+\infty}{\longrightarrow}+\infty .
$$

Remark 4.2 In particular, the solution of Problem (4.1) with periodic boundary conditions (4.5) does not become asymptotically periodic.

Proof. Note that formula (4.2) reads

$$
q(t, x)=\frac{i}{2 L} \sum_{m \in \mathbb{Z}} k_{m} \sin \left(k_{m} x\right) e^{-i k_{m}^{2} t} \tilde{f}_{0}\left(t, k_{m}\right), \quad k_{m}=\frac{m \pi}{L} .
$$

As in Section 2.3, using (4.5), we derive that

$$
\tilde{f}_{0}(t, k)=\frac{b+i k^{2} e^{i k^{2} t} \sin (b t)-b e^{i k^{2} t} \cos (b t)}{b^{2}-k^{4}} \quad \text { and } \quad \tilde{g}_{0}(t, k)=0 .
$$

Hence,

$$
\tilde{f}_{0}\left(t, \pm \frac{\pi}{L}\right)=L^{2} \frac{1-e^{2 i \frac{\pi^{2}}{L^{2}} t}}{4 \pi^{2}}+i \frac{t}{2},
$$

and for all $m \in \mathbb{Z}$ such that $|m| \geq 2$,

$$
\tilde{f}_{0}\left(t, m \frac{\pi}{L}\right)=\frac{L^{2}}{\pi^{2}} \frac{1+e^{i m^{2} \frac{\pi^{2}}{L^{2}} t}\left(i m^{2} \sin \left(\frac{\pi^{2}}{L^{2}} t\right)-\cos \left(\frac{\pi^{2}}{L^{2}} t\right)\right)}{1-m^{4}} .
$$

Using Lemma 4.3, we get that $\|q(t)\|_{L^{2}(0, L)}^{2}$ is the sum of

$$
\frac{1}{2} \frac{\pi^{2}}{L^{2}}\left(\left|\tilde{f}_{0}\left(t, k_{-1}\right)\right|^{2}+\left|\tilde{f}_{0}\left(t, k_{1}\right)\right|^{2}+\tilde{f}_{0}\left(t, k_{-1}\right) \overline{\tilde{f}_{0}\left(t, k_{1}\right)}+\tilde{f}_{0}\left(t, k_{1}\right) \overline{\tilde{f}_{0}\left(t, k_{-1}\right)}\right),
$$

and

$$
\frac{1}{2}\left(\sum_{|m| \geq 2}\left|k_{m} \tilde{f}_{0}\left(t, k_{m}\right)\right|^{2}-\sum_{|m| \geq 2} k_{m} \tilde{f}_{0}\left(t, k_{m}\right) \overline{k_{m} \tilde{f}_{0}\left(t,-k_{m}\right)}\right) .
$$

Using Hölder's inequality, we have that the real number (4.10) is non-negative. Moreover, since for all $k \in \mathbb{C}, \tilde{f}_{0}(t, k)=\tilde{f}_{0}(t,-k)$, we have that the real number (4.9) is equal to

$$
2 \frac{\pi^{2}}{L^{2}}\left|\tilde{f}_{0}\left(t, k_{1}\right)\right|^{2}
$$

Finally, (4.7) implies that $\left|\tilde{f}_{0}\left(t, k_{1}\right)\right| \underset{t \rightarrow+\infty}{\longrightarrow}+\infty$. Hence, $\|q(t)\|_{L^{2}(0, L)} \underset{t \rightarrow+\infty}{\longrightarrow}+\infty$, and so $\|q(t)\|_{L^{\infty}(0, L)} \underset{t \rightarrow+\infty}{\longrightarrow}+\infty$.

Lemma 4.3 For all sequences of complex numbers $\left(a_{k}\right)_{k \in \mathbb{Z}}$ such that $\sum_{k \in \mathbb{Z}}\left|a_{k}\right|^{2}<$ $+\infty$, the series

$$
\sum_{k \in \mathbb{Z}} a_{k} \sin \left(k \pi \frac{x}{L}\right)
$$


converges in $L^{2}(0, L)$ and its sum $f$ satisfies

$$
\frac{1}{L} \int_{0}^{L}|f(x)|^{2} \mathrm{~d} x=\frac{1}{2} \sum_{k \in \mathbb{Z}}\left|a_{k}\right|^{2}-\frac{1}{2} \sum_{k \in \mathbb{Z}} a_{k} \overline{a_{-k}} .
$$

Proof. Essentially, the proof is based on the identity valid for all $k_{1}, k_{2} \in \mathbb{Z}$ :

$$
\frac{1}{L} \int_{0}^{L} \sin \left(k_{1} \pi \frac{x}{L}\right) \sin \left(k_{2} \pi \frac{x}{L}\right) \mathrm{d} x=\frac{1}{2}\left(\delta_{k_{1}, k_{2}}-\delta_{k_{1},-k_{2}}\right),
$$

where $\delta$ is the Kronecker's symbol (i.e. $\delta_{a, b}=1$ if $a=b$ and $\delta_{a, b}=0$ otherwise).

\subsubsection{The general dependent case}

In this subsection, we still assume that $T$ and $L^{2} / \pi$ are linearly dependent on $\mathbb{Q}$. Since Problem (4.1) is linear and homogeneous, one can easily deduce the asymptotic behaviour of the general case (where both $f_{0}$ and $g_{0}$ are non-zero) from the one where $f_{0} \equiv 0$ or $g_{0} \equiv 0$. Of course, while doing so, one has to keep in mind that the possible explosions as $t \rightarrow+\infty$ (see Theorem 4.1) arising from each boundary datum can interact in a destructive way and indeed lead to a bounded solution. Consider for example the case $f_{0}(t)=g_{0}(t)=\sin (b t)$ with $b=\pi^{2} / L^{2}=2 \pi / T$. In that case, $N\left(t, k_{ \pm 1}\right)=0$ and for $|m| \geq 2, N\left(t, k_{m}\right)$ is a $2 L^{2} / \pi$-periodic function of $t$ (see (4.8)). Therefore, $q$ is itself a $2 L^{2} / \pi$-periodic function of $t$.

Hence, we can focus without too much loss of generality on the asymptotic behaviour of the solution of (4.1) with $g_{0} \equiv 0$. Any linear relation on $\mathbb{Q}$ between $T$ and $L^{2} / \pi$ yields a relation of the form

$$
\alpha \frac{2 \pi}{T}+\beta \frac{\pi^{2}}{L^{2}}=0
$$

with $(\alpha, \beta) \in(\mathbb{Z} \backslash\{0\})^{2}$ relatively primes, $\alpha \geq 1, \beta \leq-1$. Such a couple $(\alpha, \beta)$ is uniquely determined. Note that the hypotheses of Theorem 4.1 correspond to $(\alpha, \beta)=(1,-1)$.

Theorem 4.4 Let $T>0$ denote a period of the smooth function $f_{0}$, and set $g_{0} \equiv 0$. Define $b=2 \pi / T$. Denote $\left(\hat{f}_{n}\right)_{n \in \mathbb{Z}}$ the Fourier coefficients of $f_{0}$ :

$$
\forall t \in \mathbb{R}, \quad f_{0}(t)=\sum_{n \in \mathbb{Z}} \hat{f}_{n} e^{i n b t}
$$

Assume that $T$ and $L^{2} / \pi$ are linearly dependent on $\mathbb{Q}$ so that (4.11) holds. Let us denote

$$
R=\left\{(n, m) \in \mathbb{Z} \times \mathbb{Z} \quad \mid \alpha m^{2}-\beta n=0\right\} .
$$

If there exists $(n, m) \in R$ such that $\hat{f}_{n} \neq 0$, then the solution $q$ of Problem (4.1) satisfies

$$
\|q(t)\|_{L^{\infty}(0, L)} \underset{t \rightarrow+\infty}{\longrightarrow}+\infty
$$


and hence is not asymptotically periodic. Otherwise, $q$ is a periodic function of period $\left(2 L^{2} / \pi\right) \times \max (1,-\alpha / \beta)$.

Proof. Formula (4.6) can be rewritten

$$
q(t, x)=\frac{i}{L} \sum_{m \geq 1} k_{m} \sin \left(k_{m} x\right) e^{-i k_{m}^{2} t} \tilde{f}_{0}\left(t, k_{m}\right), \quad k_{m}=\frac{m \pi}{L} .
$$

Note that for all $(n, m) \in \mathbb{Z}^{2}$,

$$
((n, m) \in R) \Longleftrightarrow\left(k_{m}^{2}+n b=0\right) .
$$

For all $m \geq 1$, all $n \in \mathbb{Z}$ and all $t \geq 0$,

$$
\int_{0}^{t} e^{i k_{m}^{2} s} e^{i b n s} \mathrm{~d} s=\left\{\begin{array}{cl}
\frac{e^{i k_{m}^{2} t} e^{i n b t}-1}{i\left(k_{m}^{2}+n b\right)} & \text { if }(n, m) \notin R \\
t & \text { if }(n, m) \in R .
\end{array}\right.
$$

Note that, since $f_{0}$ is smooth, the coefficients $\hat{f}_{n}$ tend to zero rapidly as $|n| \rightarrow$ $+\infty$. Using (4.12), we write

$$
q(t, x)=q_{p}(t, x)+q_{r}(t, x),
$$

where

$$
q_{p}(t, x)=\frac{i}{L} \sum_{m \geq 1} k_{m} \sin \left(k_{m} x\right) e^{-i k_{m}^{2} t} \sum_{n \in \mathbb{Z} \mid(n, m) \notin R} \hat{f}_{n} \frac{e^{i k_{m}^{2} t} e^{i n b t}-1}{i\left(k_{m}^{2}+n b\right)},
$$

and

$$
q_{r}(t, x)=t \frac{i}{L} \sum_{m \geq 1} k_{m} \sin \left(k_{m} x\right) e^{-i k_{m}^{2} t} \sum_{n \in \mathbb{Z} \mid(n, m) \in R} \hat{f}_{n} .
$$

Note that $q_{p}$ is a periodic function and $\left(2 L^{2} / \pi\right) \times \max (1,-\alpha / \beta)$ is one if its periods. In particular, $\left\|q_{p}(t)\right\|_{L^{2}(0, L)}$ is bounded. Moreover, for all $m \geq 1$, $\sum_{n \in \mathbb{Z} \mid(n, m) \in R} \hat{f}_{n}$ contains at most 1 term. Using Lemma 4.3, we derive that

$$
\frac{1}{L} \int_{0}^{L}\left|q_{r}(t, x)\right|^{2} \mathrm{~d} x=\frac{t^{2}}{2 L^{2}} \sum_{k \geq 1} k_{m}^{2}\left|\sum_{n \in \mathbb{Z} \mid(n, m) \in R} \hat{f}_{n}\right|^{2} .
$$

This proves the result.

\subsection{The independent case}

In this section, we consider the long time behaviour of the solution $q$ of Problem (4.1) on $(0, L)$ with $g_{0} \equiv 0$ and $f_{0}$ a $T$-periodic smooth function such that $T$ and $L^{2} / \pi$ are linearly independent on $\mathbb{Q}$. Under these assumptions, we shall prove that $q$ is not asymptotically periodic (see Section 4.1 for a definition) in general (see Theorem 4.9).

In order to prove this result, we recall the following: 
Theorem 4.5 (Kronecker's approximation theorem) For all $n \in \mathbb{N}^{\star}$, for all $\left(\alpha_{1}, \ldots, \alpha_{n}\right) \in \mathbb{R}^{n}$, for all $\varepsilon, T>0$ and all linearly independent real numbers $\theta_{1}, \ldots, \theta_{n}$, there exists a real number $t>T$ and $n$ integers $h_{1}, \ldots, h_{n}$ such that

$$
\forall i \in\{1, \ldots, n\}, \quad\left|t \theta_{i}-h_{i}-\alpha_{i}\right|<\varepsilon .
$$

See [1], Theorem 7.9 and Exercise 7 of Chapter 7 for a proof of this result.

Theorem 4.6 Assume that $f, g$ and $h$ are periodic complex functions defined on $\mathbb{R}$ with

$$
h=f+g .
$$

Assume that $f$ and $g$ have positive smallest periods. We denote these periods by $T_{1}$ and $T_{2}$ respectively. Assume that $T_{1}$ and $T_{2}$ are linearly independent on $\mathbb{Q}$. Assume that $f$ is bounded. Then $f$ does not have any open interval of continuity.

See [11], Theorem 5 for a proof of this result.

\subsubsection{Preliminary results}

Lemma 4.7 Assume that $f, g$ and $h$ are periodic complex-valued functions. Let us denote respectively $T_{1}, T_{2}$ and $T_{3}$ any of their positive periods. Assume that $f$ and $g$ are continuous functions on $\mathbb{R}$ and that the function

$$
d(t)=f(t)+g(t)-h(t),
$$

tends to 0 when $t$ tends to $+\infty$. Then $h$ is continuous on $\mathbb{R}$.

Proof. Assume $\varepsilon>0$ and $t_{0} \in \mathbb{R}$ are given. Since $d(t) \underset{t \rightarrow+\infty}{\longrightarrow} 0$ and $T_{3}>0$, there exists $n \in \mathbb{N}$ such that

$$
\forall s \geq t_{0}-1, \quad\left|d\left(s+n T_{3}\right)\right|<\varepsilon / 4 .
$$

We infer that for all $s \geq t_{0}-1$,

$$
\begin{aligned}
\left|h\left(t_{0}\right)-h(s)\right| & =\left|h\left(t_{0}+n T_{3}\right)-h\left(s+n T_{3}\right)\right| \\
& \leq\left|f\left(t_{0}+n T_{3}\right)-f\left(s+n T_{3}\right)\right|+\left|g\left(t_{0}+n T_{3}\right)-g\left(s+n T_{3}\right)\right|+\varepsilon / 2 .
\end{aligned}
$$

Since $f$ and $g$ are continuous at $t_{0}+n T_{3}$, we derive that there exists $\eta>0$ such that for all $s \in\left(t_{0}-\eta, t_{0}+\eta\right)$,

$$
\left|f\left(t_{0}+n T_{3}\right)-f\left(s+n T_{3}\right)\right|<\varepsilon / 4 \quad \text { and } \quad\left|g\left(t_{0}+n T_{3}\right)-g\left(s+n T_{3}\right)\right|<\varepsilon / 4 \text {. }
$$

Hence, for all $s \in \mathbb{R}$ such that $\left|s-t_{0}\right|<\min (1, \eta)$, we have

$$
\left|h\left(t_{0}\right)-h(s)\right| \leq \varepsilon \text {. }
$$

Therefore, $h$ is continuous in $t_{0}$.

The next theorem states that, if a sum of two continuous periodic functions with uncommensurable smallest positive periods is asymptotically periodic, then it is periodic. 
Theorem 4.8 Assume that $f, g$ and $h$ are periodic complex-valued functions defined on $\mathbb{R}$. Assume that $f$ and $g$ are continuous on $\mathbb{R}$ and that they admit smallest positive periods denoted by $T_{1}$ and $T_{2}$ respectively. Assume that $T_{1}$ and $T_{2}$ are linearly independent on $\mathbb{Q}$ and that the function

$$
d(t)=f(t)+g(t)-h(t),
$$

tends to 0 when $t$ tends to $+\infty$. Then $d \equiv 0$. In other words,

$$
\forall t \in \mathbb{R}, \quad f(t)+g(t)=h(t) .
$$

Proof. Let $T_{3}$ denote any of the positive periods of $h$. Using Lemma 4.7, the function $h$ is continuous on $\mathbb{R}$. Since $f, g$ and $h$ are periodic continuous functions on $\mathbb{R}$, they are uniformly continuous on $\mathbb{R}$. Since $T_{1}$ and $T_{2}$ are positive $\mathbb{Q}$-linearly independent real numbers, so are $1 / T_{1}$ and $1 / T_{2}$. This proof is divided in two parts, depending on whether $1 / T_{3} \in \operatorname{Span}_{\mathbb{Q}}\left(1 / T_{1}, 1 / T_{2}\right)$ or not.

Assume that $1 / T_{3} \in \operatorname{Span}_{\mathbb{Q}}\left(1 / T_{1}, 1 / T_{2}\right)$.

There exists $a, b, c \in \mathbb{Z}$ such that

$$
a \frac{1}{T_{3}}=b \frac{1}{T_{2}}+c \frac{1}{T_{1}},
$$

and one can assume without loss of generality that $a>0$. Hence,

$$
a T_{1} T_{2}=b T_{1} T_{3}+c T_{2} T_{3} .
$$

Fix $s \in \mathbb{R}$. Assume $\varepsilon>0$ is given. Since $d(t) \underset{t \rightarrow+\infty}{\longrightarrow} 0$ and $a T_{1} T_{2}>0$, we can choose $T>0$ such that

$$
\forall t>T, \quad\left|d\left(s+t a T_{1} T_{2}\right)\right|<\varepsilon .
$$

Moreover, since $f, g$ and $h$ are uniformly continuous on $\mathbb{R}$, there exists $\eta>0$ such that for all $t, u \in \mathbb{R}$ with $|t-u|<\eta$, one has

$$
|f(t)-f(u)|<\varepsilon \quad \text { and } \quad|g(t)-g(u)|<\varepsilon \quad \text { and } \quad|h(t)-h(u)|<\varepsilon \text {. }
$$

Since $1 / T_{1}$ and $1 / T_{2}$ are linearly independent, so are $T_{1}$ and $T_{2}$. Hence, the Kronecker's approximation theorem (Theorem 4.5) ensures that there exists $t>$ $T$ and $x_{1}, x_{2} \in \mathbb{Z}$ such that

$$
\left|t T_{1}-x_{1}\right|<\frac{\eta}{\max \left(a T_{2},|b| T_{3},|c| T_{3}\right)} \quad \text { and } \quad\left|t T_{2}-x_{2}\right|<\frac{\eta}{\max \left(a T_{1},|b| T_{3},|c| T_{3}\right)} \text {. }
$$

Relation (4.14) ensures that

$$
d(s)=d\left(s+a t T_{1} T_{2}\right)+\delta_{t}^{1}(s)+\delta_{t}^{2}(s)-\delta_{t}^{3}(s),
$$

where

$$
\delta_{t}^{1}(s)=f(s)-f\left(s+a t T_{1} T_{2}\right), \quad \delta_{t}^{2}(s)=g(s)-g\left(s+a t T_{1} T_{2}\right),
$$

and

$$
\delta_{t}^{3}(s)=h(s)-h\left(s+a t T_{1} T_{2}\right) .
$$


Since $f$ is $T_{1}$-periodic, we have

$$
\delta_{t}^{1}(s)=f\left(s+a x_{2} T_{1}\right)-f\left(s+a t T_{1} T_{2}\right) .
$$

Moreover, using (4.19), we have

$$
\left|\left(s+a x_{2} T_{1}\right)-\left(s+a t T_{1} T_{2}\right)\right|=a T_{1}\left|x_{2}-t T_{2}\right|<\eta .
$$

Hence, using (4.18) and (4.20), we have

$$
\left|\delta_{t}^{1}(s)\right|<\varepsilon .
$$

Similarly, we have

$$
\delta_{t}^{2}(s)=g\left(s+a x_{1} T_{2}\right)-g\left(s+a t T_{1} T_{2}\right),
$$

and, using (4.19),

$$
\left|\left(s+a x_{1} T_{2}\right)-\left(s+a t T_{1} T_{2}\right)\right|=a T_{2}\left|x_{1}-t T_{1}\right|<\eta,
$$

and hence

$$
\left|\delta_{t}^{2}(s)\right|<\varepsilon .
$$

In order to get a bound on $\delta_{t}^{3}(s)$, we note that, using (4.16), we have

$$
a t T_{1} T_{2}=b t T_{1} T_{3}+c t T_{2} T_{3} .
$$

Therefore, using (4.21),

$$
\delta_{t}^{3}(s)=h(s)-h\left(s+b T_{3} t T_{1}+c T_{3} t T_{2}\right) .
$$

Hence, defining

$$
\delta_{t}^{4}(s)=h(s)-h\left(s+b T_{3} x_{1}+c T_{3} t T_{2}\right),
$$

and

$$
\delta_{t}^{5}(s)=h\left(s+b T_{3} x_{1}+c T_{3} t T_{2}\right)-h\left(s+b T_{3} t T_{1}+c T_{3} t T_{2}\right),
$$

we have,

$$
\delta_{t}^{3}(s)=\delta_{t}^{4}(s)+\delta_{t}^{5}(s) .
$$

Note that, since $h$ is $T_{3}$-periodic and $b, c, x_{1}, x_{2} \in \mathbb{Z}$,

$$
\delta_{t}^{4}(s)=h\left(s+\left(b x_{1}+c x_{2}\right) T_{3}\right)-h\left(s+b T_{3} x_{1}+c T_{3} t T_{2}\right) .
$$

Using (4.18) and (4.19), we derive that

$$
\left|\delta_{t}^{4}(s)\right|<\varepsilon \quad \text { and } \quad\left|\delta_{t}^{5}(s)\right|<\varepsilon .
$$

This yields

$$
\left|\delta_{t}^{3}(s)\right|<2 \varepsilon .
$$

We derive from the inequalities (4.17), (4.23), (4.24) and (4.25) that

$$
|d(s)|<5 \varepsilon \text {. }
$$

Since $s \in \mathbb{R}$ and $\varepsilon>0$ are arbitrary, this proves that (4.15) holds true when $1 / T_{3} \in \operatorname{Span}_{\mathbb{Q}}\left(1 / T_{1}, 1 / T_{2}\right)$.

Assume now that $1 / T_{3} \notin \operatorname{Span}_{\mathbb{Q}}\left(1 / T_{1}, 1 / T_{2}\right)$. 
In that case, $1 / T_{1}, 1 / T_{2}$ and $1 / T_{3}$ are linearly independent on $\mathbb{Q}$. After multiplication by $T_{1} T_{2} T_{3}$, this implies that $T_{1} T_{2}, T_{2} T_{3}$ and $T_{1} T_{3}$ are linearly independent on $\mathbb{Q}$. Assume $\varepsilon>0$ is given. We choose $\eta>0$ as we did in (4.18) from the uniform continuity of $f, g$ and $h$ on $\mathbb{R}$. Fix $s \in \mathbb{R}$. As before, since $d(t) \underset{t \rightarrow+\infty}{\longrightarrow} 0$, we can choose $T>0$ big enough to ensure that $\left|d\left(s+t T_{1} T_{2} T_{3}\right)\right|<\varepsilon$. Using Kronecker's approximation theorem (Theorem 4.5), we get the existence of $t>T$ and $\left(x_{1}, x_{2}, x_{3}\right) \in \mathbb{Z}^{3}$ such that

$\left|t T_{1} T_{2}-x_{3}\right|<\eta / T_{3}, \quad\left|t T_{1} T_{3}-x_{2}\right|<\eta / T_{2}, \quad$ and $\quad\left|t T_{2} T_{3}-x_{1}\right|<\eta / T_{1}$.

Using (4.14), we derive that

$$
d(s)=d\left(s+t T_{1} T_{2} T_{3}\right)+\tilde{\delta}_{t}^{1}(s)+\tilde{\delta}_{t}^{2}(s)-\tilde{\delta}_{t}^{3}(s)
$$

with

$$
\tilde{\delta}_{t}^{1}(s)=f(s)-f\left(s+t T_{1} T_{2} T_{3}\right), \quad \tilde{\delta}_{t}^{2}(s)=g(s)-g\left(s+t T_{1} T_{2} T_{3}\right),
$$

and

$$
\tilde{\delta}_{t}^{3}=h(s)-h\left(s+t T_{1} T_{2} T_{3}\right) .
$$

From (4.26), we derive that $\left|t T_{1} T_{2} T_{3}-x_{1} T_{1}\right|<\eta$, so that

$$
\left|\tilde{\delta}_{t}^{1}(s)\right|=\left|f(s)-f\left(s+t T_{1} T_{2} T_{3}\right)\right|=\left|f\left(s+x_{1} T_{1}\right)-f\left(s+t T_{1} T_{2} T_{3}\right)\right|<\varepsilon,
$$

using the $T_{1}$-periodicity of $f$. Similarly, we have

$$
\left|\tilde{\delta}_{t}^{2}(s)\right|=\left|g(s)-g\left(s+t T_{1} T_{2} T_{3}\right)\right|=\left|g\left(s+x_{2} T_{2}\right)-g\left(s+t T_{1} T_{2} T_{3}\right)\right|<\varepsilon,
$$

and

$$
\left|\tilde{\delta}_{t}^{3}(s)\right|=\left|h(s)-h\left(s+t T_{1} T_{2} T_{3}\right)\right|=\left|h\left(s+x_{2} T_{2}\right)-h\left(s+t T_{1} T_{2} T_{3}\right)\right|<\varepsilon .
$$

Using the triangle inequality in (4.27), we obtain

$$
|d(s)|<4 \varepsilon .
$$

This proves (4.15) when $1 / T_{3} \notin \operatorname{Span}_{\mathbb{Q}}\left(1 / T_{1}, 1 / T_{2}\right)$.

\subsubsection{Long time asymptotics in the independent case}

Theorem 4.9 Let us denote by $q$ the smooth solution of problem (4.1) corresponding to a smooth periodic boundary datum $f_{0}$ with smallest period $T>0$ and $g_{0} \equiv 0$. Assume that $T$ and $L^{2} / \pi$ are linearly independent on $\mathbb{Q}$. For all $x \in(0, L)$, the function $t \mapsto q(t, x)$ is not asymptotically periodic.

See Section 4.1 for a definition of asymptotic periodicity.

Proof. For all $t>0$, we have, using (4.2)

$$
q(t, x)=\frac{i}{2 L} \sum_{k \in \mathbb{Z}} k_{m} \sin \left(k_{m} x\right) e^{-i k_{m}^{2} t} \tilde{f}_{0}\left(t, k_{m}\right),
$$

in $L^{2}(0, L)$, where $\tilde{f}_{0}$ is defined in $(4.3)$. Since $f_{0}$ is $T$-periodic, we write the 
Fourier expansion of $f_{0}$, setting as before $b=2 \pi / T$ :

$$
\forall t \in \mathbb{R}, \quad f_{0}(t)=\sum_{n \in \mathbb{Z}} \hat{f}_{n} e^{i n b t} .
$$

Since $T$ and $L^{2} / \pi$ are $\mathbb{Q}$-linearly independent, we have for all $m, n \in \mathbb{Z}$

$$
k_{m}^{2}+n b=\frac{\pi^{2}}{L^{2}} m^{2}+n \frac{2 \pi}{T}=\frac{\pi^{2}}{T L^{2}}\left(m^{2} T+2 n \frac{L^{2}}{\pi}\right) \neq 0 .
$$

We derive that for all $m \in \mathbb{Z}$,

$$
\tilde{f}_{0}\left(t, k_{m}\right)=\sum_{n \in \mathbb{Z}} \hat{f}_{n} \frac{e^{i k_{m}^{2} t} e^{i n b t}-1}{i\left(k_{m}^{2}+b n\right)} .
$$

Hence, using (4.28), we can define the functions

$$
f_{x}(t)=\frac{1}{2 L} \sum_{k \in \mathbb{Z}} k_{m} \sin \left(k_{m} x\right)\left(\sum_{n \in \mathbb{Z}} \hat{f}_{n} \frac{e^{i n b t}}{k_{m}^{2}+n b}\right),
$$

and

$$
g_{x}(t)=-\frac{1}{2 L} \sum_{k \in \mathbb{Z}} k_{m} \sin \left(k_{m} x\right)\left(\sum_{n \in \mathbb{Z}} \hat{f}_{n} \frac{e^{-i k_{m}^{2} t}}{k_{m}^{2}+n b}\right),
$$

to have the following decomposition of the function $q$ :

$$
\forall x \in(0, L), \forall t \in \mathbb{R}, \quad q(t, x)=f_{x}(t)+g_{x}(t) .
$$

Note that $f_{x}$ is $T$-periodic and $g_{x}$ is $2 L^{2} / \pi$-periodic so that $q$ is an almost periodic function (see [2] and [3] for a definition). Note that, since $f_{0}$ is smooth, $f_{x}$ is a smooth function of time. Since $q$ is also a smooth function of time, so is $g_{x}$. Assume that for all $x \in(0, L)$ there exists a continuous periodic profile $q_{p}(t, x)$ such that

$$
d_{x}(t)=f_{x}(t)+g_{x}(t)-q_{p}(t, x) \underset{t \rightarrow+\infty}{\longrightarrow} 0 .
$$

Fix $x_{0} \in(0, L)$. Since $T$ and $2 L^{2} / \pi$ are linearly independent on $\mathbb{Q}$, so are the smallest positive periods of the continuous functions $f_{x_{0}}$ and $g_{x_{0}}$. Hence, Theorem 4.8 ensures that $d \equiv 0$. This yields

$$
\forall t \in \mathbb{R}, \quad f_{x_{0}}(t)+g_{x_{0}}(t)=q_{p}\left(t, x_{0}\right) .
$$

Finally, Theorem 4.6 ensures that $f_{x_{0}}$ does not have any open interval of continuity. This is a contradiction. We derive that $t \mapsto q\left(t, x_{0}\right)$ is not asymptotically periodic. 


\section{$5 \quad$ Numerical experiments}

\subsection{Linear IBVP on the half-line}

\subsubsection{The Schrödinger equation}

We first compute the numerical solution $q$ of the homogeneous linear initial boundary value problem $(2.1)$ on the half-line, with the boundary datum $f_{0}=\sin$. This fits the framework of Section 2.3 with $b=1$ in formula (2.9).

For any given positive values of $t$ and $x$, the numerical value of $q(t, x)$ is computed by integrating the integrand in relation (2.8) on the line $\gamma_{1}+\gamma_{3}$ (see $(2.15),(2.16))$. Since this function and all its derivatives have exponentially decaying moduli, we truncate the integral to a bounded interval with an error of at most $\varepsilon / 2$ where $\varepsilon$ is a given positive (small) given real number. Then, we use the trapezoidal method to compute an approximation of the integral on the bounded interval with sufficiently many steps to ensure an error of at most $\varepsilon / 2$.

We plot the results obtained for $t \in[0,20]$ and $s \in[0,7]$ on Figure 1 (real part of $q$ ) and Figure 2 (imaginary part of $q$ ).

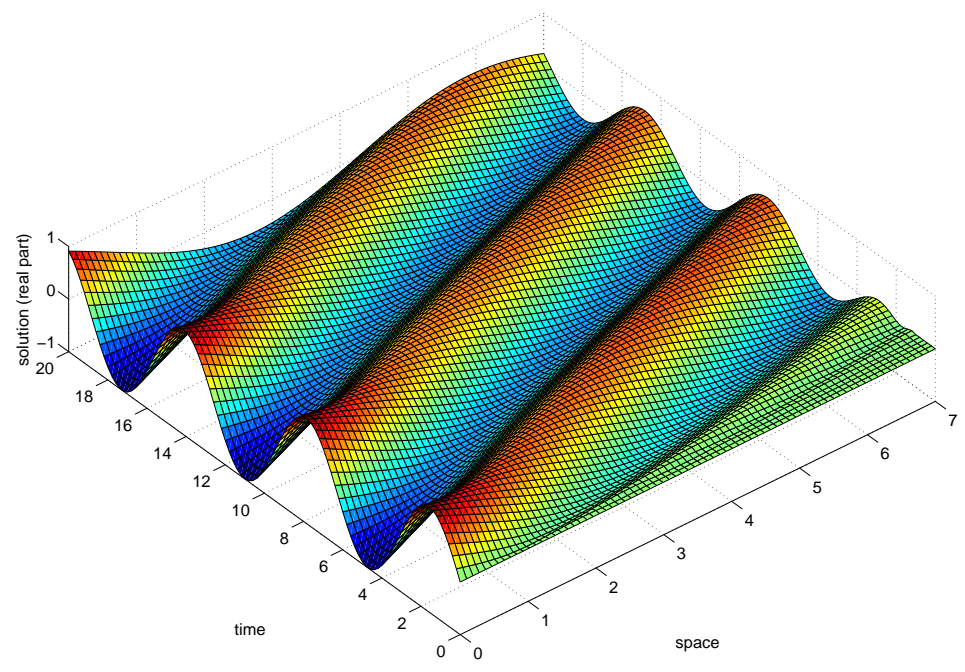

Figure 1: Real part of the solution of Problem (2.1) with boundary datum (2.9) and $b=1$.

In order to illustrate the convergence of the solution $q$ to the periodic profile provided by Theorem 2.4 and Theorem 2.14, we plot the real part of $q$ and of the profile on Figure 3 at point $x=2.0$ as functions of time. Of course, the numerical values of $q$ are computed as before. 


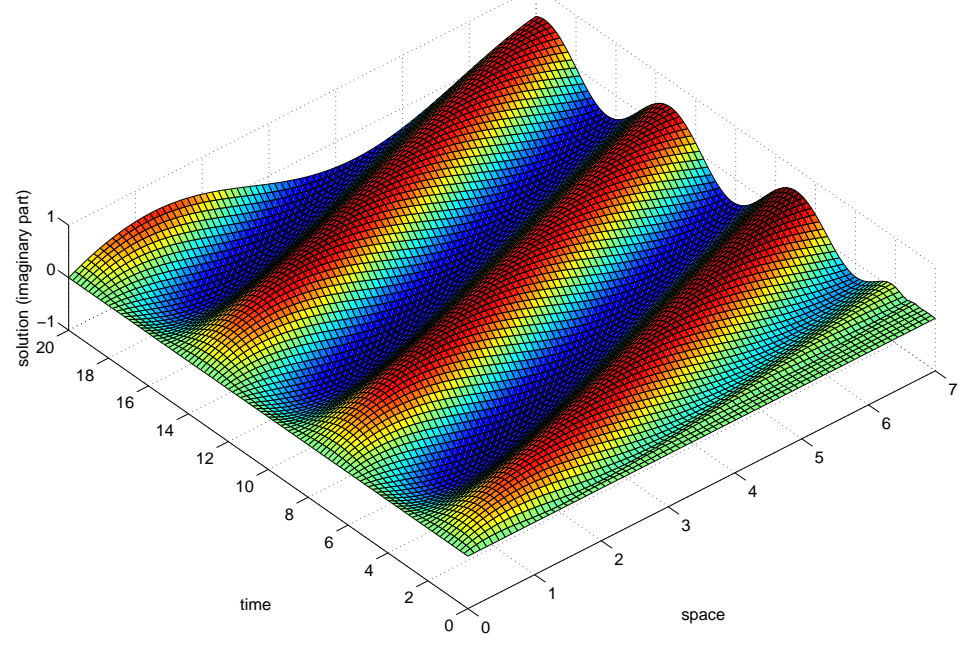

Figure 2: Imaginary part of the solution of Problem (2.1) with boundary datum (2.9) and $b=1$.

\subsubsection{The heat equation}

We now compute the numerical solution $q$ of the homogeneous linear initial boundary value problem (3.1) on the half-line, with the boundary datum $f_{0}=\sin$. This fits the framework of Section 3.1.

To compute numerical values for $q$, we use contour deformation just as we did for the Schröginger equation above. We plot the solution we obtain on Figure 4 for $t \in[0,20]$ and $x \in[0,7]$.

In order to illustrate the convergence of the solution $q$ to the periodic profile provided by Theorem 3.5, we plot $q$ and the profile on Figure 5 at point $x=2.0$ as functions of time.

\subsubsection{The linearised $\mathrm{KdV}$ equation}

As a last illustration for the asymptotics of linear IBVP problems on the half-line with periodic boundary data, we compute the solution $q$ of the linear problem (3.5) with the boundary datum

$$
\forall t \geq 0, \quad f_{0}(t)=\sin (t)+\frac{1}{10} \sin (10 t)
$$

On Figure 6, we plot the numerical results obtained for $t \in[0,30]$ and $x \in$ $[0,4]$.

In order to illustrate the convergence of the solution $q$ to the periodic profile provided by Theorem 3.6, we plot $q$ and the profile on Figure 7 at point $x=2.0$ as functions of time. 


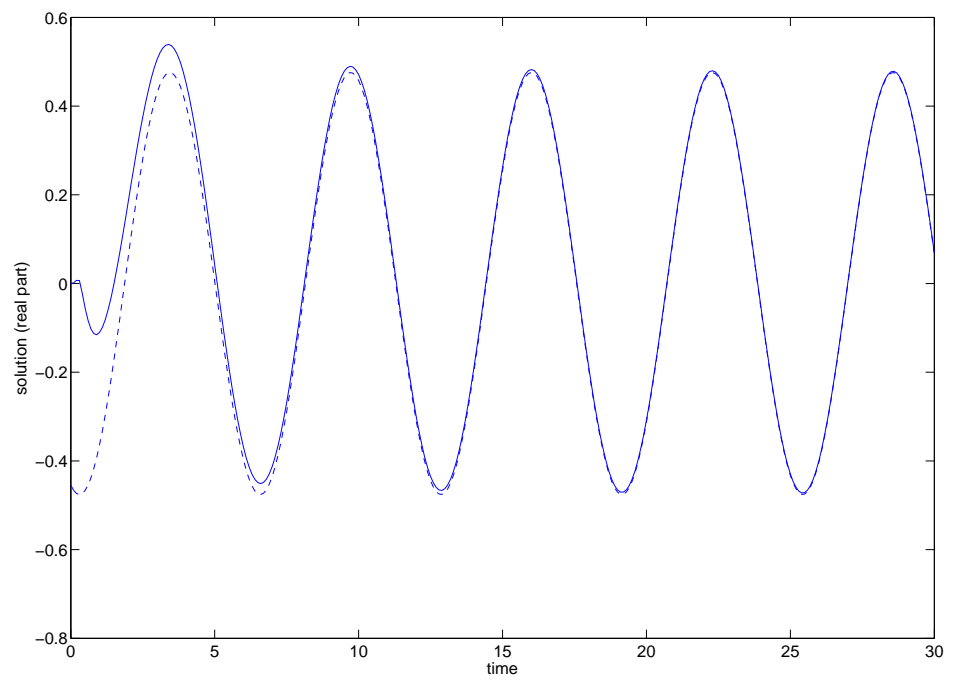

Figure 3: Solution of Problem (2.1) at point $x=2.0$ with boundary datum (2.9) and $b=1$ (full line) and the asymptotic profile given by Theorem 2.14 (dotted line).

\subsection{The linear Schrödinger equation on bounded in- tervals}

\subsubsection{The dependent case}

We investigate the asymptotic behaviour of the solutions of the IBVP (4.1) on the finite interval $(0, L)$ with $L=\pi$. We compute the numerical values of the solution $q$ using a finite difference method scheme in both time and space, with a CFL number 0.05 and 50 discretisation points in space.

Firstly, we consider periodic boundary data $f_{0}$ and $g_{0}$ defined in (4.5) with $b=1$ (i.e. $T=2 \pi$ ). Note that relation (4.4) holds true. We plot on Figure 8 the numerical values of $\|q\|_{L^{2}(0, L)}$ as a function of time for $t \in[0,50]$. We observe that the $L^{2}$-norm of $q$ is equivalent to a linear function of time in that case and hence tends to $+\infty$. This is consistent with Theorem 4.1. Note that, with the notations of Theorem 4.4, we have $\alpha=1$ and $\beta=-1$ in that case. Moreover, $f_{0}$ is $2 \pi$-periodic and its only non-zero Fourier coefficients are $\hat{f}_{ \pm 1}= \pm 1 /(2 i)$. Hence, $(1,-1) \in R$ and $\hat{f}_{-1} \neq 0$. Therefore, the numerical results of Figure 8 also illustrate part of Theorem (4.4).

Secondly, we consider periodic boundary data $f_{0}$ and $g_{0}$ defined in (4.5) with $b=2$ (i.e. $T=\pi)$. Note that relation (4.11) holds, with $\alpha=1$ and $\beta=-2$. The only non-zero Fourier coefficients of $f_{0}$ are $\hat{f}_{ \pm 1}= \pm 1 /(2 i)$ (see their definition in Theorem 4.4). Hence, for all $(n, m) \in R, \hat{f}_{n}=0$. Theorem 4.4 ensures that $q$ is a periodic function of time in that case, with period $2 \pi$. This is illustrated 


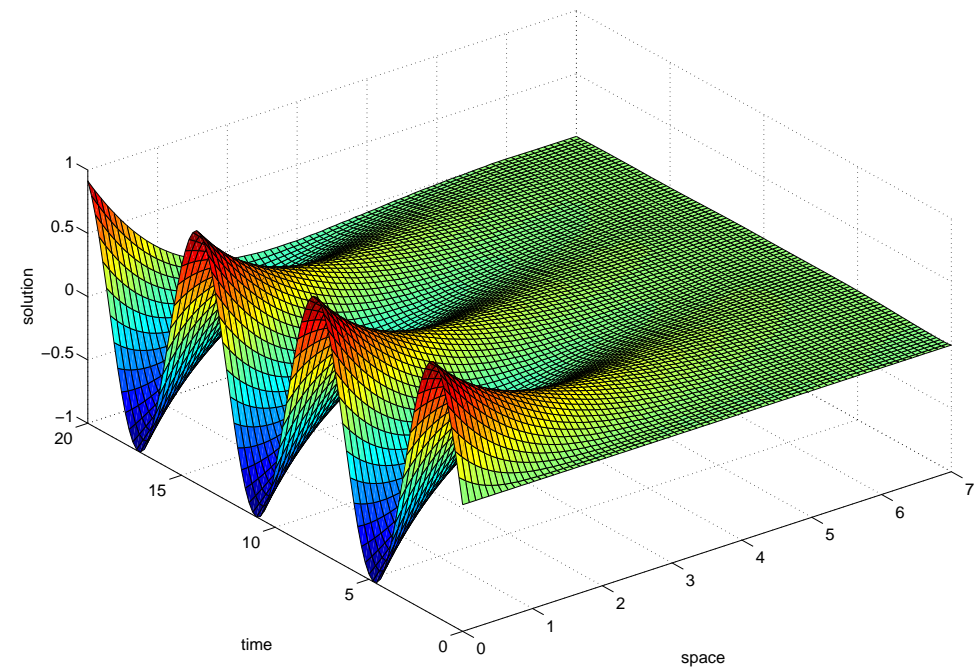

Figure 4: Solution of Problem (3.1) with boundary datum (2.9) and $b=1$.

by Figure 9 where the numerical values of $\|q\|_{L^{2}(0, L)}$ are plotted as a function of time for $t \in[0,50]$ in that case.

\subsubsection{The independent case}

We investigate the asymptotic behaviour of the solutions of the IBVP (4.1) on the finite interval $(0, L)$ with $L=1$. We compute the numerical values of the solution $q$ using a finite difference method scheme in both time and space, with a CFL number 0.05 and 50 discretisation points in space.

We consider the boundary data $f_{0}$ and $g_{0}$ given by (4.5) with $b=1$ (i.e $T=2 \pi)$. Theorem 4.9 ensures that the solution $q$ is not asymptotically periodic in that case. This is illustrated on Figure 10 where the $L^{2}$-norm of $q$ is plotted as a function of time for $t \in[0,50]$. The global behaviour of the solution is illustrated on Figure 11 where the real part of the solution is plotted for $t \in[0,50]$ and $x \in[0,1]$. Moreover, we plot on Figure 12 the real part of $q(t, x)$ evaluated at $x=0.5$ as a function of time. Figure 10, Figure 11 and Figure 12 show that, even if the solution has some stability property (the $L^{2}$-norm seems to remain bounded in that case), no periodic asymptotic behaviour seems to take place. This illustrates the result of Theorem 4.9.

\section{Acknowledgments}

The author thanks A.S. Fokas and P.A. Markowich for their ideas and comments on this work. This publication is based on work supported by Award No. KUK- 


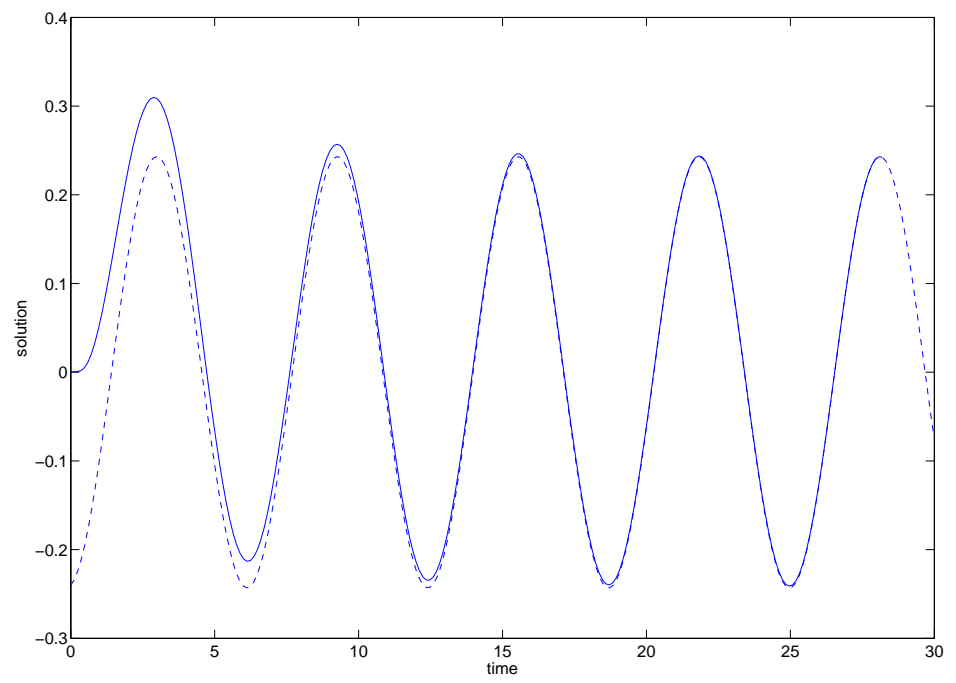

Figure 5: Solution of Problem (3.1) at point $x=2.0$ with boundary datum (2.9) and $b=1$ (full line) and the asymptotic profile given by Theorem 3.5 (dotted line).

I1-007-43, made by King Abdullah University of Science and Technology.

\section{References}

[1] T.M. Apostol , Modular functions and Dirichlet series in number theory, Springer-Verlag, 1976

[2] A.S. Besicovitch, Almost periodic functions, New-York : Chelsea Pub. Co. (1947)

[3] H.A. Bohr, Almost periodic functions, Cambridge University Press (1932)

[4] J.L. Bona, A.S. Fokas, Initial-boundary-value-problems for linear and integrable nonlinear dispersive partial differential equations, Nonlinearity 21 (2008) 195-203

[5] N. Flyer, A.S. Fokas, A hybrid analytical-numerical method for solving evolution partial differential equations. I. The half-line, Proc. R. Soc. A (2008), 464, 1823-1849

[6] A.S. Fokas, A unified transform method for solving linear and certain nonlinear PDEs, Proc. Roy. Soc. London Ser. A 453 (1997), no. 1962, 1411-1443

[7] A.S. Fokas, A new transform method for evolution PDEs, IMA J. Appl. Math. 67, 559-590 (2002)

[8] A.S. Fokas, A unified approach to boundary value problems, CBMS-NSF Regional Conference Series in Applied Mathematics, 78 (2008) 


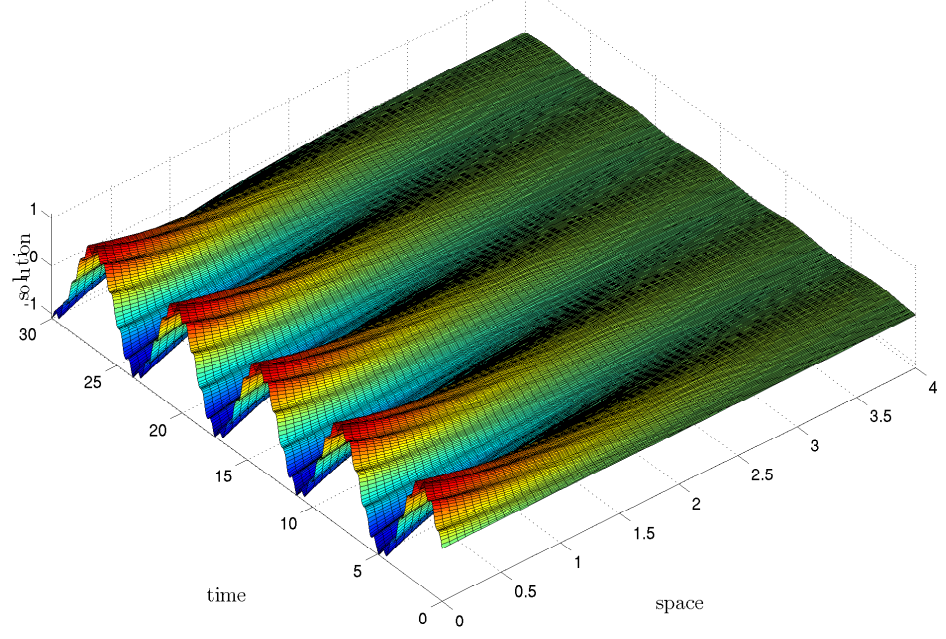

Figure 6: Solution of Problem (3.5) with boundary datum $f_{0}(t)=\sin (t)+\sin (10 t) / 10$.

[9] A.S. Fokas and B. Pelloni, A transform method for linear evolution PDEs on a finite interval, IMA J. Appl. Math. 70, 564-587 (2005)

[10] A.S. Fokas and L.-Y. Sung, Initial-boundary value problems for linear dispersive evolution equations on the half-line, Department of Mathematics, University of South Carolina. Industrial Mathematics Institute Preprint Series, 1999.

[11] J.M.H. Olmsted and C.G. Townsend, On the sum of two periodic functions, The two-year college mathematics journal, Vol. 3, No 1, Spring 1972, 33-38

[12] B. Pelloni, Well-posed boundary value problems for linear evolution equations on a finite interval, Math. Proc. Camb. Phil. Soc., 136, 361-382 


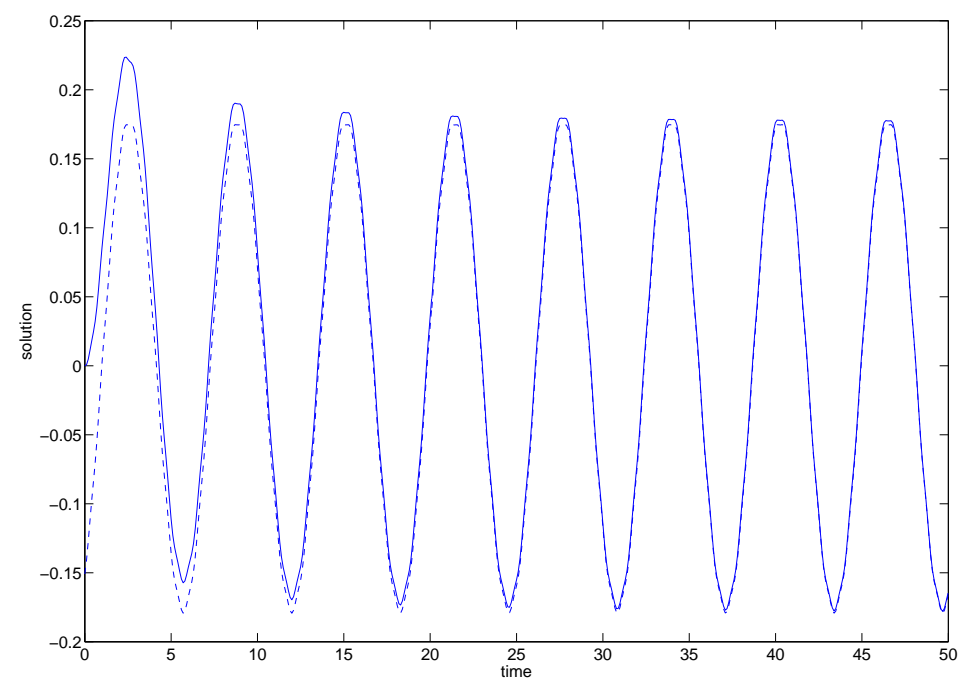

Figure 7: Solution of Problem (3.5) with boundary datum $f_{0}(t)=\sin (t)+\sin (10 t) / 10$ (full line) and the corresponding periodic profile provided by Theorem 3.6 (dotted line).

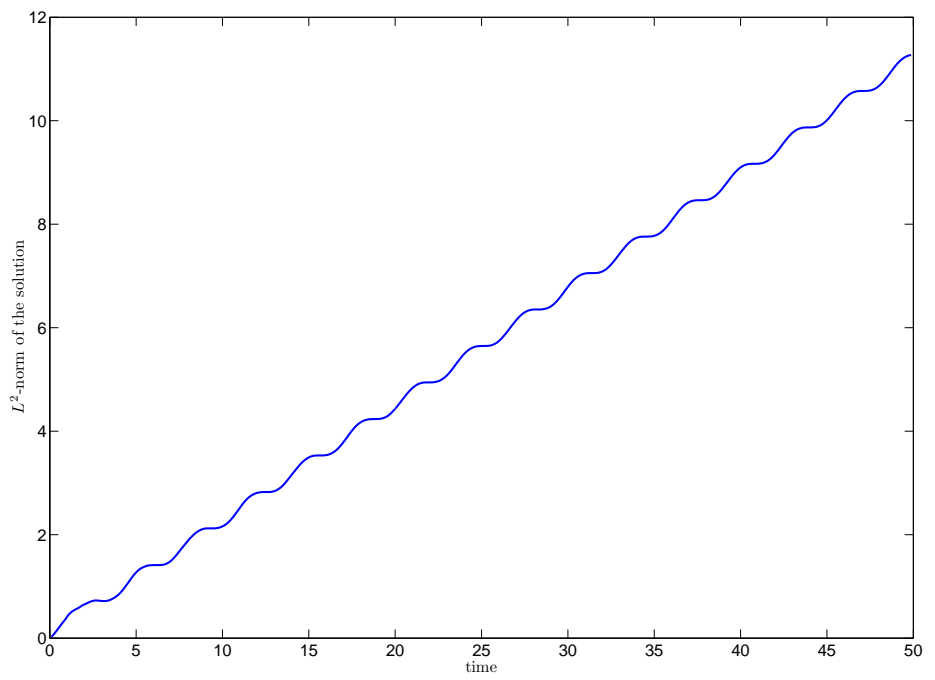

Figure 8: $L^{2}$-norm of the solution of Problem $(4.1)$ on $(0, \pi)$ with boundary data $f_{0}(t)=\sin (t)$ and $g_{0} \equiv 0$ as a function of time. 


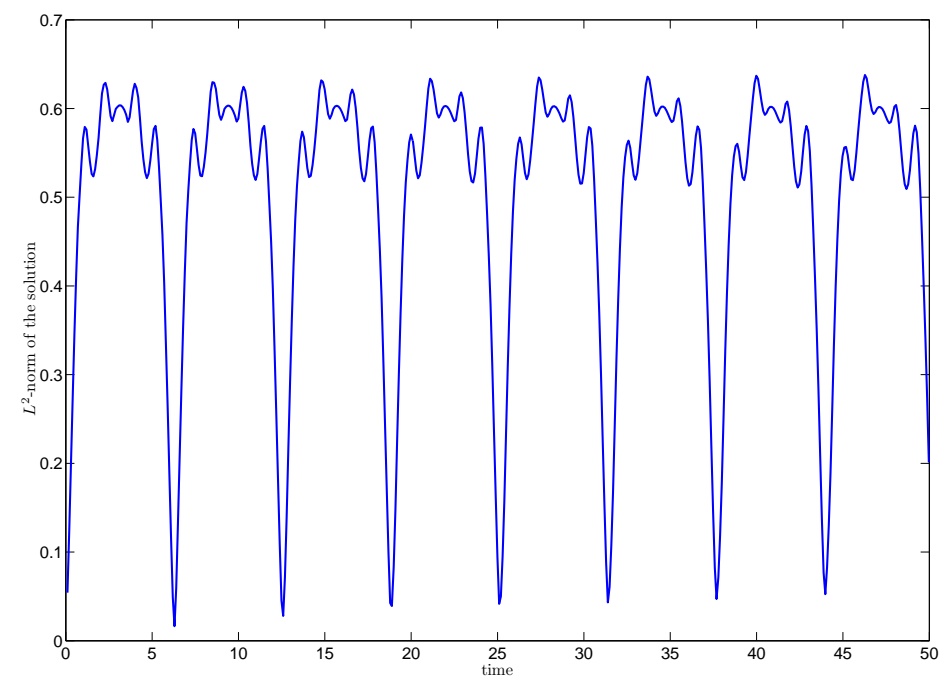

Figure 9: $L^{2}$-norm of the solution of Problem $(4.1)$ on $(0, \pi)$ with boundary data $f_{0}(t)=\sin (2 t)$ and $g_{0} \equiv 0$ as a function of time.

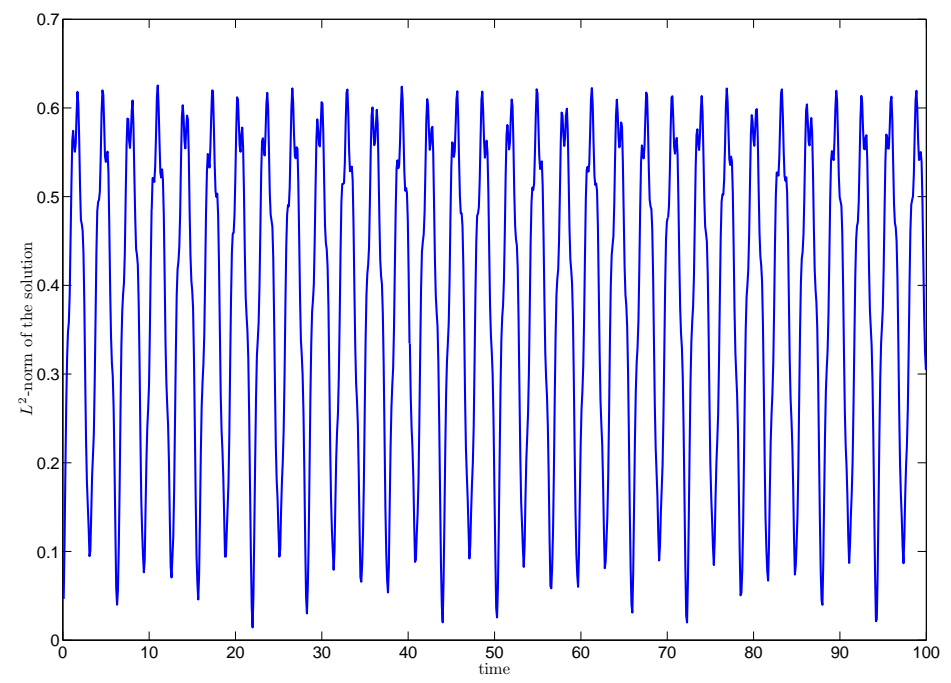

Figure 10: $L^{2}$-norm of the solution of Problem $(4.1)$ on $(0,1)$ with boundary data $f_{0}(t)=\sin (t)$ and $g_{0} \equiv 0$ as a function of time. 


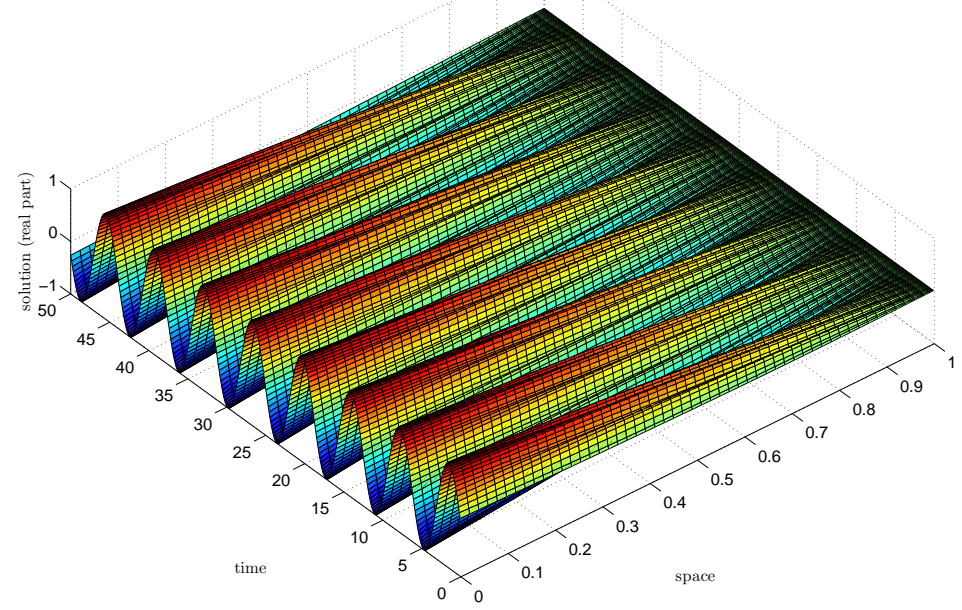

Figure 11: Real part of the solution of Problem $(4.1)$ on $(0,1)$ with boundary data $f_{0}(t)=\sin (t)$ and $g_{0} \equiv 0$.

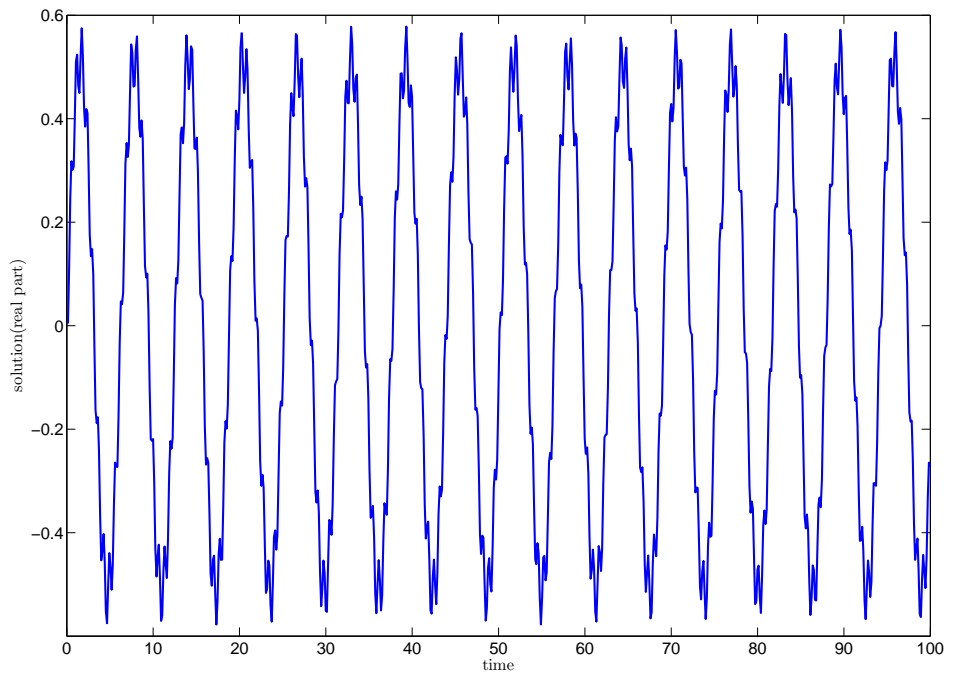

Figure 12: Real part of the solution of Problem $(4.1)$ on $(0,1)$ with boundary data $f_{0}(t)=\sin (t)$ and $g_{0} \equiv 0$ evaluated at $x=0.5$ as a function of time. 